\title{
Analytic Framework for Blended Multiple Model Systems Using Linear Local Models
}

\author{
D.J. Leith \\ W.E. Leithead \\ Department of Electronics \& Electrical Engineering, \\ University of Strathclyde, \\ 50 George St., Glasgow G1 1QE \\ Tel. +44 141548 2407, Fax: +44 141548 4203, Email: doug@icu.strath.ac.uk
}

\begin{abstract}
In this paper it is shown that the dynamics of a conventional type of blended multiple model system are only weakly related to the local models from which it is formed. A novel class of velocity-based blended multiple model systems is proposed for which the dynamics are directly related to the local models. Indeed, the solution to the blended multiple model system, locally to a specific operating point, is approximated by the weighted linear combination of the solutions to the local models. Moreover, in contrast to conventional blended multiple model systems, the velocity-based blended multiple model systems employs linear local models, thereby providing a degree of continuity with established linear methods and, consequently, facilitating analysis and design.
\end{abstract}

\section{Notation}

$\widetilde{\mathbf{x}}, \widetilde{\mathbf{w}}, \tilde{\mathbf{y}}, \tilde{\rho} \quad$ state, state velocity, output and scheduling variable of blended multiple model system (both conventional and velocity-based since no scope for confusion).

$\mathbf{r} \quad$ input.

$\mu_{\mathrm{i}} \quad$ validity function associated with $\mathrm{i}^{\text {th }}$ local model.

$\overline{\mathbf{x}}_{\mathrm{i}}, \overline{\mathbf{w}}_{\mathrm{i}}, \overline{\mathbf{y}}_{\mathrm{i}} \quad$ state, state velocity and output of $\mathrm{i}^{\text {th }}$ local model.

$\breve{\mathbf{x}}, \breve{\mathbf{w}}, \breve{\mathbf{y}} \quad$ state, state velocity and output of first-order series expansion (also perturbation forms $\delta \breve{\mathbf{x}}, \delta \breve{\mathbf{y}})$.

$\mathbf{x}, \mathbf{w}, \mathbf{y}, \rho \quad$ state, state velocity, output and scheduling variable of general nonlinear system.

$\breve{\mathbf{x}}^{*}, \breve{\mathbf{w}}^{*}, \breve{\mathbf{y}}^{*} \quad$ weighted linear combinations of solutions to velocity-based local models.

$\widetilde{\mathbf{x}}_{\mathrm{c}}, \widetilde{\mathbf{w}}_{\mathrm{c}}, \mathbf{r}_{\mathrm{c}}, \tilde{\mathbf{y}}_{\mathrm{c}}$ state, state velocity, input and output of velocity-based blended multiple controller.

$\mathbf{y}_{\text {ref }} \quad$ reference input to controller.

$\overline{\mathbf{x}}_{\mathrm{c}_{\mathrm{i}}}, \overline{\mathbf{w}}_{\mathrm{c}_{\mathrm{i}}}, \overline{\mathbf{y}}_{\mathrm{c}_{\mathrm{i}}}$ state, state velocity and output of $\mathrm{i}^{\text {th }}$ local controller.

$\varepsilon_{\mathrm{w}^{*}}, \varepsilon_{\mathrm{y}^{*}}, \varepsilon_{\mathrm{w}}$ residual terms.

subscript $_{1}$ denotes evaluation at some specific operating point; for example, $\left(\mathbf{x}_{1}, \mathbf{r}_{1}\right)$.

\section{Introduction}

Under quite general conditions, the solution to a nonlinear system approximates the solution to another nonlinear system over any finite time interval provided the terms in the differential equations describing the two systems are sufficiently similar (see, for example, Khalil 1992 Theorem 2.5). Hence, by employing a sufficiently accurate representation of the terms in the differential equation describing a nonlinear system, a nonlinear system may be obtained which is an approximation to the original system. The representation of the nonlinear mappings associated with differential equations describing nonlinear systems is currently receiving considerable attention in the literature (see, for example, the review by Johansen \& Murray-smith 1997). However, a common difficulty is the so-called "curse of dimensionality" whereby the number of parameters in the representation increases extremely rapidly as the order of the nonlinear dynamic system increases. This strongly motivates the investigation of representations which can accurately describe high order nonlinear systems with a relatively small number of parameters.

One promising class of representations is blended multiple model systems wherein a small number of relatively simple dynamic systems are, in some sense, blended together. Blended multiple model systems 
have been studied in a number of quite diverse fields (Johansen \& Murray-Smith 1997). In the context of fuzzy inference systems, Takagi \& Sugeno (1985) consider the approximate decomposition of an algebraic nonlinear mapping into a set of fuzzy rules each of the form "IF system is in operating region i THEN output is <affine function of inputs>". Whilst Takagi \& Sugeno (1985) restrict consideration to linear-type membership functions and to algebraic mappings, the extension to more general forms of membership function and to dynamic systems is straightforward (see, for example, Johansen \& Murray-Smith 1997). In the context of neural networks, Johansen \& Foss (1993) consider the approximation of the discrete-time nonlinear dynamic system, $\mathbf{y}(\mathrm{t})=\mathbf{F}(\psi(\mathrm{t}-1))+\mathbf{e}(\mathrm{t}) \quad$ with $\psi(\mathrm{t}-1)=\left[\mathbf{y}^{\mathrm{T}}(\mathrm{t}-1), \mathbf{y}^{\mathrm{T}}(\mathrm{t}-2), \ldots, \mathbf{u}^{\mathrm{T}}(\mathrm{t}-1), \mathbf{u}^{\mathrm{T}}(\mathrm{t}-2), \ldots, \mathbf{e}^{\mathrm{T}}(\mathrm{t}-\right.$ $\left.1), \mathbf{e}^{\mathrm{T}}(\mathrm{t}-2), \ldots\right]^{\mathrm{T}}$, by a local model network of the form, $\mathbf{y}(\mathrm{t})=\sum_{\mathrm{i}} \tilde{\mathbf{F}}_{\mathrm{i}}(\psi(\mathrm{t}-1)) \tilde{\mathbf{w}}_{i}(\psi(\mathrm{t}-1))+\mathbf{e}(\mathrm{t})$, where $\mathbf{y}$ is the output, $\mathbf{u}$ and $\mathbf{e}$ are the inputs, $\mathbf{F}$ and $\tilde{\mathbf{w}}_{i}$ are smooth nonlinear functions, and $\tilde{\mathbf{F}}_{i}$ are affine local models. Gawthrop (1995) considers the approximation of a continuous-time nonlinear dynamic system, in the vicinity of the equilibrium operating points, by a continuous-time local model network.

Typically, each simple system is a local model which describes the dynamics of the nonlinear system in some small region of the operating space. The role of the blending is to provide smooth interpolation, in some sense, between the local models with the aim of achieving an accurate representation with only a small number of local models. The blending is, therefore, central to the utility of the approach. Since the operating regions, in which no single local model dominates and several local models contribute significantly to the blended multiple model system, constitutes the greater part of the operating space, it is the investigation of the characteristics of the blended multiple model system in these regions that is of primary concern and which is the subject of the present paper. Whilst central to the utility of blended multiple model representations, this issue is largely neglected in the literature (with the notable exception of Shorten et al. 1998). It should be noted that another motivation for adopting a blended multiple model systems is to support a divide and conquer philosophy whereby the operating space of a nonlinear system is decomposed into operating regions within which the dynamics are described by a particular model. Each model in the blended multiple model system is valid in an extended operating region and blending is confined to small transition regions at the boundaries between these main operating regions. In this context, blending plays a relatively minor role and the approach is more akin to piecewise approximation. In this paper, consideration is confined to the former situation; that is, to blended multiple model systems where blending is employed to interpolate between a small number of local models and for which the operating regions where a number of local models are blended together include the greater part of the operating space.

The paper is organised as follows. In section 2, the conventional type of blended multiple model system is investigated. In section 3, the velocity-based linearisation approach proposed in Leith \& Leithead (1998a) is briefly reviewed and a novel class of velocity-based blended multiple model systems is proposed and analysed. An example is presented in section 4 and the conclusions are summarised in section 5.

\section{Blended multiple model systems}

Consider the conventional type of blended multiple model system

$$
\begin{array}{ll}
\dot{\tilde{\mathbf{x}}}=\sum_{\mathrm{i}} \overline{\mathbf{F}}_{\mathrm{i}}(\tilde{\mathbf{x}}, \mathbf{r}) \mu_{\mathrm{i}}(\tilde{\boldsymbol{\rho}}), \quad \tilde{\mathbf{y}}=\sum_{\mathrm{i}} \overline{\mathbf{G}}_{\mathrm{i}}(\tilde{\mathbf{x}}, \mathbf{r}) \mu_{\mathrm{i}}(\tilde{\boldsymbol{\rho}}) \\
\tilde{\rho}(\tilde{\mathbf{x}}, \mathbf{r})=\nabla_{\mathbf{x}} \tilde{\boldsymbol{\rho}} \tilde{\mathbf{x}}+\nabla_{\mathbf{r}} \tilde{\boldsymbol{\rho}} \mathbf{r} &
\end{array}
$$

where, $\tilde{\mathbf{x}} \in \mathfrak{R}^{\mathrm{n}}, \mathbf{r} \in \mathfrak{R}^{\mathrm{m}}, \tilde{\mathbf{y}} \in \mathfrak{R}^{\mathrm{p}}, \tilde{\rho}(\tilde{\mathbf{x}}, \mathbf{r}) \in \mathfrak{R}^{\mathrm{q}}$. The $\overline{\mathbf{F}}_{\mathrm{i}}$ and $\overline{\mathbf{G}}_{\mathrm{i}}$ are differentiable nonlinear functions with Lipschitz continuous first derivatives. The $\mu_{\mathrm{i}}$ are differentiable mappings, with Lipschitz continuous first derivatives, from the operating space, $\hat{\Phi} \subseteq \mathfrak{R}^{\mathrm{n}} \times \mathfrak{R}^{\mathrm{m}}$, onto the positive real line such that $\sum_{\mathrm{i}} \mu_{\mathrm{i}}(\tilde{\rho})=1$. The quantity, $\rho(\mathbf{x}, \mathbf{r})$, embodying the dependence of the blending on the state and input, is assumed to be linear in the state, $\widetilde{\mathbf{x}}$, and input, $\mathbf{r}$ : no loss of generality is involved since any nonlinear dependence can be absorbed into the validity functions, $\mu_{\mathrm{i}}$. It is assumed, in addition, that the input and initial conditions are restricted such that the solution to (1) lies within the operating space, $\hat{\Phi}$. This class of blended multiple model systems is similar to that considered by, for example, Gawthrop (1995) and Shorten et al. (1998) 
and is closely related to the systems considered by Johansen \& Foss (1993), albeit in a continuous-time rather than discrete-time setting.

The local models associated with the blended multiple model system are

$$
\dot{\overline{\mathbf{x}}}_{\mathrm{i}}=\overline{\mathbf{F}}_{\mathrm{i}}\left(\overline{\mathbf{x}}_{\mathrm{i}}, \mathbf{r}\right), \quad \overline{\mathbf{y}}_{\mathrm{i}}=\overline{\mathbf{G}}_{\mathrm{i}}\left(\overline{\mathbf{x}}_{\mathrm{i}}, \mathbf{r}\right)
$$

Typically (Johansen \& Foss 1993, Johansen \& Murray-smith 1997), affine local models are employed; that is,

$$
\overline{\mathbf{F}}_{\mathrm{i}}(\overline{\mathbf{x}}, \mathbf{r})=\bar{\alpha}_{\mathrm{i}}+\overline{\mathbf{A}}_{\mathrm{i}} \overline{\mathbf{x}}+\overline{\mathbf{B}}_{\mathrm{i}} \mathbf{r}, \quad \overline{\mathbf{G}}_{\mathrm{i}}(\overline{\mathbf{x}}, \mathbf{r})=\overline{\boldsymbol{\beta}}_{\mathrm{i}}+\overline{\mathbf{C}}_{\mathrm{i}} \overline{\mathbf{x}}+\overline{\mathbf{D}}_{\mathrm{i}} \mathbf{r}
$$

where $\bar{\alpha}_{\mathrm{i}}, \overline{\mathbf{A}}_{\mathrm{i}}, \overline{\mathbf{B}}_{\mathrm{i}}, \overline{\boldsymbol{\beta}}_{\mathrm{i}}, \overline{\mathbf{C}}_{\mathrm{i}}, \overline{\mathbf{D}}_{\mathrm{i}}$ are constants. It should be noted that, when the blended multiple model system describes a smooth nonlinear system, a corresponding smoothness requirement is imposed on the righthand sides in (1) which, for a particular choice of the validity functions, constrains the choice of local models. This constraint can be included during learning/identification by, for example, the classical regularisation approach of Tikhonov \& Arsensin (1977), originally developed in the context of optimisation theory, whereby the identification cost function is augmented with a penalty term which is related to the non-smoothness of the blended multiple model system.

To determine the dynamic behaviour of the blended multiple model system, (1), locally to an operating point, $\left(\tilde{\mathbf{x}}_{1}, \mathbf{r}_{1}\right)$, consider the first-order series expansion of (1) relative to the operating point,

$$
\begin{aligned}
& \delta \dot{\overline{\mathbf{x}}}=\sum_{\mathrm{i}} \overline{\mathbf{F}}_{\mathrm{i}}\left(\tilde{\mathbf{x}}_{1}, \mathbf{r}_{1}\right) \mu_{\mathrm{i}}\left(\tilde{\rho}_{1}\right)+\sum_{\mathrm{i}}\left(\nabla_{\mathbf{x}} \overline{\mathbf{F}}_{\mathrm{i}}\left(\tilde{\mathbf{x}}_{1}, \mathbf{r}_{1}\right) \mu_{\mathrm{i}}\left(\tilde{\rho}_{1}\right)+\overline{\mathbf{F}}_{\mathrm{i}}\left(\tilde{\mathbf{x}}_{1}, \mathbf{r}_{1}\right) \nabla \mu_{\mathrm{i}}\left(\tilde{\rho}_{1}\right) \nabla_{\mathbf{x}} \tilde{\rho}\right) \delta \tilde{\mathbf{x}}+\sum_{\mathrm{i}}\left(\nabla_{\mathbf{r}} \overline{\mathbf{F}}_{\mathrm{i}}\left(\tilde{\mathbf{x}}_{1}, \mathbf{r}_{1}\right) \mu_{\mathrm{i}}\left(\tilde{\rho}_{1}\right)+\overline{\mathbf{F}}_{\mathrm{i}}\left(\tilde{\mathbf{x}}_{1}, \mathbf{r}_{1}\right) \nabla \mu_{\mathrm{i}}\left(\tilde{\rho}_{1}\right) \nabla_{\mathbf{r}} \tilde{\rho}\right) \delta \mathbf{r} \\
& \delta \breve{\mathbf{y}}=\sum_{\mathrm{i}}\left(\nabla_{\mathbf{x}} \overline{\mathbf{G}}_{\mathrm{i}}\left(\tilde{\mathbf{x}}_{\mathbf{1}}, \mathbf{r}_{1}\right) \mu_{\mathrm{i}}\left(\tilde{\rho}_{\mathbf{1}}\right)+\overline{\mathbf{G}}_{\mathrm{i}}\left(\tilde{\mathbf{x}}_{\mathbf{1}}, \mathbf{r}_{1}\right) \nabla \mu_{\mathrm{i}}\left(\tilde{\rho}_{1}\right) \nabla_{\mathbf{x}} \tilde{\rho}\right) \delta \tilde{\mathbf{x}}+\sum_{\mathrm{i}}\left(\nabla_{\mathbf{r}} \overline{\mathbf{G}}_{\mathrm{i}}\left(\tilde{\mathbf{x}}_{\mathbf{1}}, \mathbf{r}_{1}\right) \mu_{\mathrm{i}}\left(\tilde{\rho}_{\mathbf{1}}\right)+\overline{\mathbf{G}}_{\mathrm{i}}\left(\tilde{\mathbf{x}}_{\mathbf{1}}, \mathbf{r}_{1}\right) \nabla \mu_{\mathrm{i}}\left(\tilde{\rho}_{1}\right) \nabla_{\mathbf{r}} \tilde{\rho}\right) \delta \mathbf{r}
\end{aligned}
$$

where

$$
\tilde{\rho}_{1}=\nabla_{\mathbf{x}} \tilde{\rho} \tilde{\mathbf{x}}_{1}+\nabla_{\mathbf{r}} \tilde{\rho} \mathbf{r}_{1}, \quad \delta \mathbf{r}=\mathbf{r}-\mathbf{r}_{1}, \quad \breve{\mathbf{y}}=\sum_{i} \overline{\mathbf{G}}_{\mathrm{i}}\left(\tilde{\mathbf{x}}_{1}, \mathbf{r}_{1}\right) \mu_{\mathrm{i}}\left(\tilde{\rho}_{1}\right)+\delta \breve{\mathbf{y}}, \quad \breve{\mathbf{x}}=\delta \breve{\mathbf{x}}+\tilde{\mathbf{x}}_{1}, \dot{\overline{\mathbf{x}}}=\delta \dot{\overline{\mathbf{x}}}
$$

It should be noted that the expansion is carried out relative to the fixed operating point, $\left(\widetilde{\mathbf{x}}_{1}, \mathbf{r}_{1}\right)$, as opposed to a trajectory passing through $\left(\tilde{\mathbf{x}}_{1}, \mathbf{r}_{1}\right)$, and the derivative of $\tilde{\mathbf{x}}_{1}$ is, therefore, zero. It is emphasised that (5)-(7) is a well-defined system in its own right, distinct from the nonlinear system (1), with state $\delta \widetilde{\mathbf{x}}$. Furthermore, the point, $\left(\widetilde{\mathbf{x}}_{1}, \mathbf{r}_{1}\right)$, need not be an equilibrium operating point and, indeed, may lie far from the locus of equilibrium operating points. When $\left(\tilde{\mathbf{x}}_{1}, \mathbf{r}_{1}\right)$ is an equilibrium operating point, the inhomogeneous term in (5) is zero in which case the dynamics of (5)-(7) are determined solely by the terms in $\delta \breve{\mathbf{x}}$ and $\delta \mathbf{r}$ (note that, in general, the contributions to these terms from both $\mu_{\mathrm{i}}$ and $\nabla \mu_{\mathrm{i}}$ are significant).

However, when $\left(\tilde{\mathbf{x}}_{\mathbf{1}}, \mathbf{r}_{\mathbf{1}}\right)$ is a non-equilibrium operating point the inhomogeneous term may be extremely large and can dominate the solution to (5)-(7).

Combining (5) and (6) with the local input, output and state transformations, (7), the first-order representation, (5)-(7), may be reformulated as

$$
\begin{aligned}
& \dot{\tilde{\mathbf{x}}}=\sum_{\mathrm{i}} \overline{\mathbf{F}}_{\mathrm{i}}\left(\tilde{\mathbf{x}}_{1}, \mathbf{r}_{1}\right) \mu_{\mathrm{i}}\left(\tilde{\rho}_{1}\right)-\sum_{\mathrm{i}}\left(\nabla_{\mathrm{x}} \overline{\mathbf{F}}_{\mathrm{i}}\left(\tilde{\mathbf{x}}_{1}, \mathbf{r}_{1}\right) \mu_{\mathrm{i}}\left(\tilde{\rho}_{1}\right)+\overline{\mathbf{F}}_{\mathrm{i}}\left(\tilde{\mathbf{x}}_{1}, \mathbf{r}_{1}\right) \nabla \mu_{\mathrm{i}}\left(\tilde{\rho}_{1}\right) \nabla_{\mathrm{x}} \tilde{\rho}\right) \tilde{\mathbf{x}}_{1}-\sum_{\mathrm{i}}\left(\nabla_{\mathrm{r}} \overline{\mathbf{F}}_{\mathrm{i}}\left(\tilde{\mathbf{x}}_{1}, \mathbf{r}_{1}\right) \mu_{\mathrm{i}}\left(\tilde{\rho}_{1}\right)+\overline{\mathbf{F}}_{\mathrm{i}}\left(\tilde{\mathbf{x}}_{1}, \mathbf{r}_{1}\right) \nabla \mu_{\mathrm{i}}\left(\tilde{\rho}_{1}\right) \nabla_{\mathrm{r}} \tilde{\rho}\right) \mathbf{r}_{1} \\
& +\sum_{\mathrm{i}}\left(\nabla_{\mathrm{x}} \overline{\mathbf{F}}_{\mathrm{i}}\left(\tilde{\mathbf{x}}_{1}, \mathbf{r}_{1}\right) \mu_{\mathrm{i}}\left(\tilde{\rho}_{1}\right)+\overline{\mathbf{F}}_{\mathrm{i}}\left(\tilde{\mathbf{x}}_{1}, \mathbf{r}_{1}\right) \nabla \mu_{\mathrm{i}}\left(\tilde{\rho}_{1}\right) \nabla_{\mathrm{x}} \tilde{\rho}\right) \tilde{\mathbf{x}}+\sum_{\mathrm{i}}\left(\nabla_{\mathbf{r}} \overline{\mathbf{F}}_{\mathrm{i}}\left(\tilde{\mathbf{x}}_{1}, \mathbf{r}_{1}\right) \mu_{\mathrm{i}}\left(\tilde{\rho}_{1}\right)+\overline{\mathbf{F}}_{\mathrm{i}}\left(\tilde{\mathbf{x}}_{1}, \mathbf{r}_{1}\right) \nabla \mu_{\mathrm{i}}\left(\tilde{\rho}_{1}\right) \nabla_{\mathbf{r}} \tilde{\rho}\right) \mathbf{r} \\
& \breve{\mathbf{y}}=\sum_{\mathrm{i}} \overline{\mathbf{G}}_{\mathrm{i}}\left(\tilde{\mathbf{x}}_{1}, \mathbf{r}_{1}\right) \mu_{\mathrm{i}}\left(\tilde{\rho}_{1}\right)-\sum_{\mathrm{i}}\left(\nabla_{\mathbf{x}} \overline{\mathbf{G}}_{\mathrm{i}}\left(\tilde{\mathbf{x}}_{1}, \mathbf{r}_{1}\right) \mu_{\mathrm{i}}\left(\tilde{\rho}_{1}\right)+\overline{\mathbf{G}}_{\mathrm{i}}\left(\tilde{\mathbf{x}}_{1}, \mathbf{r}_{1}\right) \nabla \mu_{\mathrm{i}}\left(\tilde{\rho}_{1}\right) \nabla_{\mathrm{x}} \tilde{\rho}\right) \tilde{\mathbf{x}}_{1}-\sum_{\mathrm{i}}\left(\nabla_{\mathrm{r}} \overline{\mathbf{G}}_{\mathrm{i}}\left(\tilde{\mathbf{x}}_{1}, \mathbf{r}_{1}\right) \mu_{\mathrm{i}}\left(\tilde{\rho}_{1}\right)+\overline{\mathbf{G}}_{\mathrm{i}}\left(\tilde{\mathbf{x}}_{1}, \mathbf{r}_{1}\right) \nabla \mu_{\mathrm{i}}\left(\tilde{\rho}_{1}\right) \nabla_{\mathrm{r}} \tilde{\rho}\right) \mathbf{r}_{1} \\
& +\sum_{i}\left(\nabla_{\mathbf{x}} \overline{\mathbf{G}}_{\mathrm{i}}\left(\tilde{\mathbf{x}}_{\mathbf{1}}, \mathbf{r}_{1}\right) \mu_{\mathrm{i}}\left(\tilde{\rho}_{\mathbf{1}}\right)+\overline{\mathbf{G}}_{\mathrm{i}}\left(\tilde{\mathbf{x}}_{\mathbf{1}}, \mathbf{r}_{1}\right) \nabla \mu_{\mathrm{i}}\left(\tilde{\rho}_{1}\right) \nabla_{\mathrm{x}} \tilde{\rho}\right) \tilde{\mathbf{x}}+\sum_{\mathrm{i}}\left(\nabla_{\mathbf{r}} \overline{\mathbf{G}}_{\mathrm{i}}\left(\tilde{\mathbf{x}}_{\mathbf{1}}, \mathbf{r}_{1}\right) \mu_{\mathrm{i}}\left(\tilde{\rho}_{\mathbf{1}}\right)+\overline{\mathbf{G}}_{\mathrm{i}}\left(\tilde{\mathbf{x}}_{\mathbf{1}}, \mathbf{r}_{1}\right) \nabla \mu_{\mathrm{i}}\left(\tilde{\rho}_{1}\right) \nabla_{\mathbf{r}} \tilde{\rho}\right) \mathbf{r}
\end{aligned}
$$

In contrast to (5)-(7), the state, input and output are now the same at every operating point, $\left(\tilde{\mathbf{x}}_{1}, \mathbf{r}_{1}\right)$. When the local models are affine, (4), the first-order representation, (8), is

$$
\begin{aligned}
& \dot{\grave{\mathbf{x}}}=\sum_{\mathrm{i}}\left(\bar{\alpha}_{\mathrm{i}} \mu_{\mathrm{i}}\left(\tilde{\rho}_{1}\right)-\left(\bar{\alpha}_{\mathrm{i}}+\overline{\mathbf{A}}_{\mathrm{i}} \breve{\mathbf{x}}_{\mathbf{1}}+\overline{\mathbf{B}}_{\mathrm{i}} \mathbf{r}_{1}\right) \nabla \mu_{\mathrm{i}}\left(\tilde{\rho}_{1}\right) \tilde{\rho}_{1}\right) \\
& \quad+\sum_{\mathrm{i}}\left(\overline{\mathbf{A}}_{\mathrm{i}} \mu_{\mathrm{i}}\left(\tilde{\rho}_{1}\right)+\left(\bar{\alpha}_{\mathrm{i}}+\overline{\mathbf{A}}_{\mathrm{i}} \overline{\mathbf{x}}_{1}+\overline{\mathbf{B}}_{\mathrm{i}} \mathbf{r}_{1}\right) \nabla \mu_{\mathrm{i}}\left(\tilde{\rho}_{1}\right) \nabla_{\mathrm{x}} \tilde{\rho}\right) \tilde{\mathbf{x}}+\sum_{\mathrm{i}}\left(\overline{\mathbf{B}}_{\mathrm{i}} \mu_{\mathrm{i}}\left(\tilde{\rho}_{1}\right)+\left(\bar{\alpha}_{\mathrm{i}}+\overline{\mathbf{A}}_{\mathrm{i}} \overline{\mathbf{x}}_{1}+\overline{\mathbf{B}}_{\mathrm{i}} \mathbf{r}_{1}\right) \nabla \mu_{\mathrm{i}}\left(\tilde{\rho}_{1}\right) \nabla_{\mathbf{r}} \tilde{\rho}\right) \mathbf{r}
\end{aligned}
$$




$$
\begin{aligned}
\breve{\mathbf{y}}=\sum_{\mathrm{i}}\left(\overline{\boldsymbol{\beta}}_{\mathrm{i}} \mu_{\mathrm{i}}\left(\tilde{\rho}_{1}\right)-\left(\overline{\boldsymbol{\beta}}_{\mathrm{i}}+\overline{\mathbf{C}}_{\mathrm{i}} \overline{\mathbf{x}}_{\mathbf{1}}+\overline{\mathbf{D}}_{\mathrm{i}} \mathbf{r}_{1}\right) \nabla \mu_{\mathrm{i}}\left(\tilde{\rho}_{1}\right) \tilde{\rho}_{1}\right) \\
\quad+\sum_{\mathrm{i}}\left(\overline{\mathbf{C}}_{\mathrm{i}}+\left(\overline{\boldsymbol{\beta}}_{\mathrm{i}}+\overline{\mathbf{C}}_{\mathrm{i}} \overline{\mathbf{x}}_{\mathbf{1}}+\overline{\mathbf{D}}_{\mathrm{i}} \mathbf{r}_{1}\right) \nabla \mu_{\mathrm{i}}\left(\tilde{\rho}_{1}\right) \nabla_{\mathbf{x}} \tilde{\boldsymbol{\rho}}\right) \tilde{\mathbf{x}}+\sum_{\mathrm{i}}\left(\overline{\mathbf{D}}_{\mathrm{i}}+\left(\overline{\boldsymbol{\beta}}_{\mathrm{i}}+\overline{\mathbf{C}}_{\mathrm{i}} \overline{\mathbf{x}}_{\mathbf{1}}+\overline{\mathbf{D}}_{\mathrm{i}} \mathbf{r}_{1}\right) \nabla \mu_{\mathrm{i}}\left(\tilde{\rho}_{1}\right) \nabla_{\mathrm{r}} \tilde{\boldsymbol{\rho}}\right) \mathbf{r}
\end{aligned}
$$

The blending in a blended multiple model system is required to provide smooth interpolation, in some sense, between the local models to achieve an accurate representation over the whole operating space with only a small number of local models. The operating regions, in which no single local model dominates and several local models contribute significantly to the blended multiple model system, constitute the greater part of the operating space. However, to facilitate analysis and design, the dynamics of the blended multiple model system in these regions are still required to be directly related to the local models. This property, for example, underlies control design approaches such as that of Hunt \& Johansen (1997), whereby a linear controller is designed for each local model and the members of the family of linear controllers obtained are blended to obtain a nonlinear controller. Locally to the operating point, $\left(\widetilde{\mathbf{x}}_{1}, \mathbf{r}_{1}\right)$, the solution, $\tilde{\mathbf{x}}$, to the blended multiple model system is approximated by the solution, $\widetilde{\mathbf{x}}$, to the first-order series expansion (8) (Leith \& Leithead 1998a; see also section 3.1). However, it is evident from (8) that the relationship between the first-order series expansions and the local models is not straightforward. The dynamics of the blended multiple model system are not described by a simple blend of dynamics of the local models. Indeed, in operating regions where the derivative, $\nabla \mu_{\mathrm{i}}$, of any validity function is large, the dynamics of the first-order series expansions may be strongly influenced by the varying nature of the validity functions and only weakly related to the dynamics of the local models. Hence, the utility of this class of blended multiple model systems is diminished considerably. Furthermore, although widely employed in the literature, it is noted that affine local models do not provide continuity with established linear methods and, in particular, do not possess the superposition property which is fundamental to linear systems. It is emphasised that the magnitude of the inhomogeneous term in an affine local model may be extremely large and can strongly influence the solution. When the inhomogeneous term varies from one local model to another, it introduces an implicit feedback from the state and/or input of the overall blended multiple model system. Hence, the inhomogeneous term cannot, in general, simply be treated as a disturbance or small approximation error.

\section{Velocity-based blended multiple model systems}

Two difficulties with the conventional approach to blended multiple model systems using affine local models are noted above; namely, the lack of a direct relationship, between the dynamics of the blended multiple model system and the dynamics of the local models, and the lack of linearity of the local models themselves. To circumvent these difficulties the velocity-based representation of nonlinear dynamics systems of Leith \& Leithead (1998a) is adopted; that is, the dynamics are represented by a velocity-based nonlinear system or, equivalently, a velocity-based linearisation family.

\subsection{Velocity-based linearisation families}

Before proceeding, the velocity-based approach of Leith \& Leithead (1998a) is briefly reviewed. Consider nonlinear plants with dynamics,

$$
\dot{\mathbf{x}}=\mathbf{F}(\mathbf{x}, \mathbf{r}), \quad \mathbf{y}=\mathbf{G}(\mathbf{x}, \mathbf{r})
$$

where $\mathbf{F}(\cdot, \cdot)$ and $\mathbf{G}(\cdot, \cdot)$ are differentiable nonlinear functions with Lipschitz continuous first derivatives and $\mathbf{r} \in \mathfrak{R}^{\mathrm{m}}$ denotes the input to the plant, $\mathbf{y} \in \mathfrak{R}^{\mathrm{p}}$ the output and $\mathbf{x} \in \mathfrak{R}^{\mathrm{n}}$ the states. The set of equilibrium operating points of the nonlinear plant, (11), consists of those points, $\left(\mathbf{x}_{\mathbf{0}}, \mathbf{r}_{\mathbf{0}}\right)$, for which

$$
\mathbf{F}\left(\mathbf{x}_{\mathbf{0}}, \mathbf{r}_{\mathbf{o}}\right)=0
$$

and the corresponding equilibrium output is

$$
\mathbf{y}_{\mathbf{0}}=\mathbf{G}\left(\mathbf{x}_{\mathbf{0}}, \mathbf{r}_{\mathbf{0}}\right)
$$

Let $\Phi: \Re^{\mathrm{n}} \times \Re^{\mathrm{m}}$ denote the space consisting of the union of the states, $\mathbf{x}$, with the inputs, $\mathbf{r}$. The set of equilibrium operating points of the nonlinear plant, (11), forms a locus of points, $\left(\mathbf{x}_{\mathbf{0}}, \mathbf{r}_{\mathbf{0}}\right)$, in $\Phi$ and the response of the plant to a general time-varying input, $\mathbf{r}(\mathrm{t})$, is depicted by a trajectory in $\Phi$.

The nonlinear system, (11), may be reformulated, equivalently, as

$$
\dot{\mathbf{x}}=\mathbf{A x}+\mathbf{B r}+\mathbf{f}(\rho), \quad \mathbf{y}=\mathbf{C x}+\mathbf{D r}+\mathbf{g}(\rho)
$$


where $\mathbf{A}, \mathbf{B}, \mathbf{C}, \mathbf{D}$ are appropriately dimensioned constant matrices, $\mathbf{f}(\bullet)$ and $\mathbf{g}(\bullet)$ are nonlinear functions and $\rho(\mathbf{x}, \mathbf{r}) \in \mathfrak{R}^{\mathrm{q}}, \mathrm{q} \leq \mathrm{m}+\mathrm{n}$, embodies the nonlinear dependence of the dynamics on the state and input with $\nabla_{\mathbf{x}} \rho, \nabla_{\mathbf{r}} \rho$ functions of $\rho$ alone. Trivially, this reformulation can always be achieved by letting $\rho=\left[\mathbf{x}^{\mathrm{T}} \mathbf{r}^{\mathrm{T}}\right]^{\mathrm{T}}$, in which case $q=m+n$. However, the nonlinearity of the system is frequently dependent on only a subset of the states and inputs, in which case the dimension, $q$, of $\rho$ is less than $m+n$. Since $\nabla_{x} \rho, \nabla_{\mathbf{r}} \rho$ are functions of $\rho$ alone, the variable, $\rho(\mathbf{x}, \mathbf{r})$, equals the constant value, $\rho_{1}$, upon a surface of co-dimension q in $\Phi$ and $\nabla_{\mathbf{x}} \rho$ and $\nabla_{\mathbf{r}} \rho$ are constant over each surface. Hence, the normal to each surface is identical at every point on the surface and each surface is, therefore, affine. Moreover, to ensure that $\rho$ is a unique function of $\mathbf{x}$ and $\mathbf{r}$, these surfaces must be parallel for all $\rho$. Consequently, it may in fact be assumed, without loss of generality, that $\nabla_{\mathrm{x}} \rho$ and $\nabla_{\mathrm{r}} \rho$ are constant.

Suppose that the nonlinear system, (14), is evolving along a trajectory, $(\mathbf{x}(\mathrm{t}), \mathbf{r}(\mathrm{t}))$, in $\Phi$ and at time, $\mathrm{t}_{1}$, the trajectory has reached the point, $\left(\mathbf{x}_{1}, \mathbf{r}_{1}\right)$. It is emphasised that the point, $\left(\mathbf{x}_{1}, \mathbf{r}_{1}\right)$, need not be an equilibrium operating point and, indeed, may lie far from the locus of equilibrium operating points. From Taylor series expansion theory, the subsequent behaviour of the nonlinear system can be approximated, locally to the point, $\left(\mathbf{x}_{1}, \mathbf{r}_{1}\right)$, by the first order representation,

$$
\begin{aligned}
& \delta \dot{\widetilde{\mathbf{x}}}=\left(\mathbf{A} \mathbf{x}_{1}+\mathbf{B r} \mathbf{r}_{1}+\mathbf{f}\left(\rho_{1}\right)\right)+\left(\mathbf{A}+\nabla \mathbf{f}\left(\rho_{1}\right) \nabla_{\mathbf{x}} \rho\right) \delta \check{\mathbf{x}}+\left(\mathbf{B}+\nabla \mathbf{f}\left(\rho_{1}\right) \nabla_{\mathbf{r}} \boldsymbol{\rho}\right) \delta \mathbf{r} \\
& \delta \breve{\mathbf{y}}=\left(\mathbf{C}+\nabla \mathbf{g}\left(\rho_{1}\right) \nabla_{\mathbf{x}} \rho\right) \delta \breve{\mathbf{x}}+\left(\mathbf{D}+\nabla \mathbf{g}\left(\rho_{1}\right) \nabla_{\mathbf{r}} \rho\right) \delta \mathbf{r} \\
& \rho_{1}=\rho\left(\mathbf{x}_{1}, \mathbf{r}_{1}\right), \quad \delta \mathbf{r}=\mathbf{r}-\mathbf{r}_{1}, \quad \breve{\mathbf{y}}=\mathbf{C} \mathbf{x}_{1}+\operatorname{Dr}_{1}+\mathbf{g}\left(\rho_{1}\right)+\delta \breve{\mathbf{y}}, \quad \breve{\mathbf{x}}=\delta \breve{\mathbf{x}}+\mathbf{x}_{1}, \dot{\breve{\mathbf{x}}}=\delta \dot{\widetilde{\mathbf{x}}}
\end{aligned}
$$

provided $\mathbf{x}_{1}+\delta \breve{\mathbf{x}} \subseteq \mathrm{N}_{\mathbf{x}} \mathbf{r}_{1}+\delta \mathbf{r} \subseteq \mathrm{N}_{\mathbf{r}}$, where the neighbourhoods, $\mathrm{N}_{\mathbf{x}}$ and $\mathrm{N}_{\mathbf{r}}$, of, respectively, $\mathbf{x}_{\mathbf{1}}$ and $\mathbf{r}_{\mathbf{1}}$ are sufficiently small. When $\delta \breve{\mathbf{x}}\left(\mathrm{t}_{1}\right)$ is zero,

$$
\begin{aligned}
& \breve{\mathbf{x}}\left(\mathrm{t}_{1}\right)=\mathbf{x}_{1}=\mathbf{x}\left(\mathrm{t}_{1}\right) \\
& \dot{\check{\mathbf{x}}}\left(\mathrm{t}_{1}\right)=\mathbf{A} \mathbf{x}_{1}+\mathbf{B} \mathbf{r}_{1}+\mathbf{f}\left(\rho_{1}\right)=\dot{\mathbf{x}}\left(\mathrm{t}_{1}\right) \\
& \ddot{\breve{\mathbf{x}}}\left(\mathrm{t}_{1}\right)=\left(\mathbf{A}+\nabla \mathbf{f}\left(\rho_{1}\right) \nabla_{\mathbf{x}} \rho \dot{\mathbf{x}}\left(\mathrm{t}_{1}\right)\right) \dot{\breve{\mathbf{x}}}\left(\mathrm{t}_{1}\right)+\left(\mathbf{B}+\nabla \mathbf{f}\left(\rho_{1}\right) \nabla_{\mathbf{r}} \rho\right) \dot{\mathbf{r}}\left(\mathrm{t}_{1}\right)=\ddot{\mathbf{x}}\left(\mathrm{t}_{1}\right) \\
& \check{\mathbf{y}}\left(\mathrm{t}_{1}\right)=\mathbf{C \mathbf { x } _ { 1 }}+\mathbf{D \mathbf { r } _ { 1 }}+\mathbf{g}\left(\rho_{1}\right)=\mathbf{y}\left(\mathrm{t}_{1}\right) \\
& \dot{\breve{\mathbf{y}}}\left(\mathrm{t}_{1}\right)=\left(\mathbf{C}+\nabla \mathbf{g}\left(\rho_{1}\right) \nabla_{\mathbf{x}} \rho\right) \dot{\check{\mathbf{x}}}\left(\mathrm{t}_{1}\right)+\left(\mathbf{D}+\nabla \mathbf{g}\left(\rho_{1}\right) \nabla_{\mathbf{r}} \rho\right) \dot{\mathbf{r}}\left(\mathrm{t}_{1}\right)=\dot{\mathbf{y}}\left(\mathrm{t}_{1}\right)
\end{aligned}
$$

Hence, the solution, $\breve{\mathbf{x}}(\mathrm{t})$, to (15)-(17), initially at time $\mathrm{t}_{1}$, is tangential to the solution, $\mathbf{x}(\mathrm{t})$, of (14). Indeed, locally to time $\mathrm{t}_{1}, \breve{\mathbf{x}}(\mathrm{t})$ provides a first-order approximation to $\dot{\mathbf{x}}(\mathrm{t})$ and a second-order approximation to $\mathbf{x}(\mathrm{t})$ and $\breve{\mathbf{y}}(\mathrm{t})$ provides a first-order approximation to $\mathbf{y}(\mathrm{t})$.

The solution, $\breve{\mathbf{x}}(\mathrm{t})$, to the first-order series expansion, (15)-(17), provides a valid approximation only while the solution, $\mathbf{x}(\mathrm{t})$, to the nonlinear system remains in the vicinity of the operating point, $\left(\mathbf{x}_{1}, \mathbf{r}_{1}\right)$. However, the solution, $\mathbf{x}(\mathrm{t})$, to the nonlinear system need not stay in the vicinity of a single operating point. Consider the time interval, $[0, \mathrm{~T}]$; the initial time can, without loss of generality, always be taken as zero. An approximation to $\mathbf{x}(\mathrm{t})$ is obtained by partitioning the interval into a number of short sub-intervals. Over each sub-interval, the approximate solution is the solution to the first-order series expansion relative to the operating point reached at the initial time for the sub-interval (with the initial conditions for each subinterval chosen to ensure continuity of the approximate solution). The number of local solutions employed is dependent on the duration of the sub-intervals, but the local solutions are accurate to second order; that is, the approximation error is proportional to the duration of the sub-interval cubed. Hence, as the number of sub-intervals increases, the approximation error associated with each rapidly decreases and the overall approximation error also decreases. Indeed, the overall approximation error tends to zero as the maximum size of the sub-intervals tends to zero. Hence, the family of first-order series expansions, with members defined by (15)-(17), can provide an arbitrarily accurate approximation to the solution of the nonlinear system. Moreover, this approximation property holds throughout $\Phi$ and is not confined to the vicinity of a single equilibrium operating point or even of the locus of equilibrium operating points.

It should be noted that the state, input and output transformations, (17), depend on the operating point relative to which the series expansion is carried out. When the solution to the nonlinear system is confined to a neighbourhood about a single operating point, the transformations, (17), are static and the dynamic behaviour is described by the system, (15)-(16), alone. However, when the solution to the nonlinear system traces a trajectory which is not confined to a neighbourhood about a single operating point, the transformations, (17), are no longer static and the dynamic behaviour is no longer described solely by the 
system, (15)-(16). Instead, the dynamic behaviour is described by (15)-(17). Combining (15) and (16) with the local input, output and state transformations, (17), each member, (15)-(17), of the family of firstorder representations may be reformulated as,

$$
\begin{aligned}
& \dot{\overline{\mathbf{x}}}=\left\{\mathbf{f}\left(\rho_{1}\right)-\nabla \mathbf{f}\left(\rho_{1}\right) \nabla_{\mathbf{x}} \rho \mathbf{x}_{\mathbf{1}}-\nabla \mathbf{f}\left(\rho_{1}\right) \nabla_{\mathbf{r}} \rho \mathbf{r}_{1}\right\}+\left(\mathbf{A}+\nabla \mathbf{f}\left(\rho_{1}\right) \nabla_{\mathbf{x}} \rho\right) \check{\mathbf{x}}+\left(\mathbf{B}+\nabla \mathbf{f}\left(\rho_{1}\right) \nabla_{\mathbf{r}} \rho\right) \mathbf{r} \\
& \check{\mathbf{y}}=\left\{\mathbf{g}\left(\rho_{1}\right)-\nabla \mathbf{g}\left(\rho_{1}\right) \nabla_{\mathbf{x}} \rho \mathbf{x}_{\mathbf{1}}-\nabla \mathbf{g}\left(\rho_{1}\right) \nabla_{\mathbf{r}} \rho \mathbf{r}_{1}\right\}+\left(\mathbf{C}+\nabla \mathbf{g}\left(\rho_{1}\right) \nabla_{\mathbf{x}} \rho\right) \check{\mathbf{x}}+\left(\mathbf{D}+\nabla \mathbf{g}\left(\rho_{1}\right) \nabla_{\mathbf{r}} \rho\right) \mathbf{r}
\end{aligned}
$$

In contrast to the representation, (15)-(16), the state, input and output are now the same for all members of the reformulated family. The dynamics, (23)-(24), of an individual member of the family are affine rather than linear even when $\left(\mathbf{x}_{1}, \mathbf{r}_{1}\right)$ is an equilibrium operating point. The inhomogeneous terms in (23)-(24) may, in general, be extremely large and can dominate the solution.

On differentiating (23)-(24)

$$
\begin{aligned}
& \dot{\check{\mathbf{x}}}=\breve{\mathbf{w}} \\
& \dot{\breve{\mathbf{w}}}=\left(\mathbf{A}+\nabla \mathbf{f}\left(\rho_{1}\right) \nabla_{\mathbf{x}} \rho\right) \breve{\mathbf{w}}+\left(\mathbf{B}+\nabla \mathbf{f}\left(\rho_{1}\right) \nabla_{\mathbf{r}} \rho\right) \dot{\mathbf{r}} \\
& \dot{\check{\mathbf{y}}}=\left(\mathbf{C}+\nabla \mathbf{g}\left(\rho_{1}\right) \nabla_{\mathbf{x}} \rho\right) \breve{\mathbf{w}}+\left(\mathbf{D}+\nabla \mathbf{g}\left(\rho_{1}\right) \nabla_{\mathbf{r}} \rho\right) \dot{\mathbf{r}}
\end{aligned}
$$

The system, (25)-(27), is dynamically equivalent to the system, (23)-(24), in the sense that with appropriate initial conditions, namely,

$$
\breve{\mathbf{x}}\left(\mathrm{t}_{1}\right)=\mathbf{x}_{1}, \quad \breve{\mathbf{w}}\left(\mathrm{t}_{1}\right)=\mathbf{A} \mathbf{x}_{1}+\mathbf{B r} \mathbf{r}_{1}+\mathbf{f}\left(\rho_{1}\right), \quad \breve{\mathbf{y}}\left(\mathrm{t}_{1}\right)=\mathbf{C x _ { 1 }}+\operatorname{Dr}_{1}+\mathbf{g}\left(\rho_{1}\right)
$$

the solution, $\breve{\mathbf{x}}$, to (25)-(27), is the same as the solution, $\breve{\mathbf{x}}$, to (23)-(24). However, in contrast to (23)-(24) , the transformed system, (25)-(27), is linear. The relationship between the nonlinear system and its velocity-based linearisation, (25)-(27), is direct. Differentiating (14), an alternative representation of the nonlinear system is

$$
\begin{aligned}
& \dot{\mathbf{x}}=\mathbf{w} \\
& \dot{\mathbf{w}}=\left(\mathbf{A}+\nabla \mathbf{f}(\rho) \nabla_{\mathrm{x}} \rho\right) \mathbf{w}+\left(\mathbf{B}+\nabla \mathbf{f}(\rho) \nabla_{\mathrm{r}} \rho\right) \dot{\mathbf{r}} \\
& \dot{\mathbf{y}}=\left(\mathbf{C}+\nabla \mathbf{g}(\rho) \nabla_{\mathbf{x}} \rho\right) \mathbf{w}+\left(\mathbf{D}+\nabla \mathbf{g}(\rho) \nabla_{\mathrm{r}} \rho\right) \dot{\mathbf{r}}
\end{aligned}
$$

Dynamically, (29)-(31), with appropriate initial conditions corresponding to (28), and (14) are equivalent (have the same solution, $\mathbf{x})$. (When $\mathbf{w}=\mathbf{F}(\mathbf{x}, \mathbf{r}), \mathbf{y}=\mathbf{G}(\mathbf{x}, \mathbf{r})$ is invertible for every $(\mathbf{x}, \mathbf{r})$, so that $\mathbf{x}$ may be expressed as a function of $\mathbf{w}, \mathbf{r}$ and $\mathbf{y}$, then the transformation relating (29)-(31) to (14) is, in fact, algebraic). When

$$
\breve{\mathbf{x}}\left(\mathrm{t}_{1}\right)=\mathbf{x}\left(\mathrm{t}_{1}\right), \quad \breve{\mathbf{w}}\left(\mathrm{t}_{1}\right)=\mathbf{w}\left(\mathrm{t}_{1}\right), \quad \breve{\mathbf{y}}\left(\mathrm{t}_{1}\right)=\mathbf{y}\left(\mathrm{t}_{1}\right)
$$

it follows from (25)-(27) and (29)-(31) that

$$
\begin{aligned}
& \dot{\check{\mathbf{x}}}\left(\mathrm{t}_{1}\right)=\breve{\mathbf{w}}\left(\mathrm{t}_{1}\right)=\dot{\mathbf{x}}\left(\mathrm{t}_{1}\right) \\
& \ddot{\breve{\mathbf{x}}}\left(\mathrm{t}_{1}\right)=\left(\mathbf{A}+\nabla \mathbf{f}\left(\rho_{1}\right) \nabla_{\mathbf{x}} \rho \dot{\mathbf{x}}\left(\mathrm{t}_{1}\right)\right) \breve{\mathbf{w}}\left(\mathrm{t}_{1}\right)+\left(\mathbf{B}+\nabla \mathbf{f}\left(\rho_{1}\right) \nabla_{\mathbf{r}} \rho\right) \dot{\mathbf{r}}\left(\mathrm{t}_{1}\right)=\ddot{\mathbf{x}}\left(\mathrm{t}_{1}\right) \\
& \dot{\breve{\mathbf{w}}}\left(\mathrm{t}_{1}\right)=\left(\mathbf{A}+\nabla \mathbf{f}\left(\rho_{1}\right) \nabla_{\mathbf{x}} \rho \dot{\mathbf{x}}\left(\mathrm{t}_{1}\right)\right) \breve{\mathbf{w}}\left(\mathrm{t}_{1}\right)+\left(\mathbf{B}+\nabla \mathbf{f}\left(\rho_{1}\right) \nabla_{\mathbf{r}} \rho\right) \dot{\mathbf{r}}\left(\mathrm{t}_{1}\right)=\dot{\mathbf{w}}\left(\mathrm{t}_{1}\right) \\
& \dot{\dot{\mathbf{y}}}\left(\mathrm{t}_{1}\right)=\left(\mathbf{C}+\nabla \mathbf{g}\left(\rho_{1}\right) \nabla_{\mathrm{x}} \rho\right) \breve{\mathbf{w}}\left(\mathrm{t}_{1}\right)+\left(\mathbf{D}+\nabla \mathbf{g}\left(\rho_{1}\right) \nabla_{\mathbf{r}} \rho\right) \dot{\mathbf{r}}\left(\mathrm{t}_{1}\right)=\dot{\mathbf{y}}\left(\mathrm{t}_{1}\right)
\end{aligned}
$$

Hence, $\breve{\mathbf{x}}(\mathrm{t})$ and $\breve{\mathbf{y}}(\mathrm{t})$ still provide a second- and first-order approximation to, respectively, $\mathbf{x}(\mathrm{t})$ and $\mathbf{y}(\mathrm{t})$ and $\breve{\mathbf{w}}(\mathrm{t})$ provides a first-order approximation to $\mathbf{w}(\mathrm{t})$. Clearly, the velocity-based linearisation, (25)-(27), is simply the frozen form of (29)-(31) at the operating point, $\left(\mathbf{x}_{1}, \mathbf{r}_{1}\right)$.

There exists a velocity-based linearisation, (25)-(27), for every point in $\Phi$. Hence, a velocity-based linearisation family, with members defined by (25)-(27), can be associated with the nonlinear system, (14). Similarly to the family of first-order expansions, the solutions to the members of the family of velocitybased linearisations, (25)-(27), can be pieced together (with the initial conditions for each sub-interval chosen to ensure continuity of $\breve{\mathbf{x}}, \breve{\mathbf{w}}$ and $\breve{\mathbf{y}}$ ) to approximate the solution to the nonlinear system, (29)-(31) to an arbitrary degree of accuracy. However, it should be noted that, for any particular partition of $[0, \mathrm{~T}]$, the approximate solutions, obtained using the family of velocity-based linearisations and the family of firstorder expansions, differ even though both converge on the solution to (14).

There exists a rigorous, and direct, relationship between the dynamic characteristics of a nonlinear system and those of a related family of linear systems, namely, the velocity-based linearisation family, and a related family of affine systems, namely the first-order series expansion family. Since the solutions to the members of the families can be combined to approximate the solution to the nonlinear system arbitrarily accurately, the families embody the entire dynamics of the nonlinear system, (14), with no loss of information and therefore provide alternative representations of the nonlinear system. Whilst these representations are equivalent in the sense that they each embody the entire dynamics of the nonlinear 
system, they are not necessarily equivalent with respect to other considerations. In particular, the direct relationship between the velocity-form of the nonlinear system and the velocity-based linearisation family and the linearity of the members of the latter family provides continuity with established linear theory which, for example, facilitates analysis (Leith \& Leithead 1998a) and controller design (Leith \& Leithead 1998c).

\subsection{Velocity-based blended multiple model systems}

Instead of using the direct forms of the local models, consider constructing a blended multiple model system using the velocity-based forms; that is, consider the velocity-based blended multiple model system

$$
\begin{aligned}
& \dot{\tilde{\mathbf{x}}}=\tilde{\mathbf{w}} \\
& \dot{\tilde{\mathbf{w}}}=\left(\sum_{\mathrm{i}} \phi_{\mathrm{x}}^{\mathrm{i}} \mu_{\mathrm{i}}(\tilde{\rho})\right) \tilde{\mathbf{w}}+\left(\sum_{\mathrm{i}} \phi_{\mathrm{r}}^{\mathrm{i}} \mu_{\mathrm{i}}(\tilde{\boldsymbol{\rho}})\right) \dot{\mathbf{r}} \\
& \dot{\tilde{\mathbf{y}}}=\left(\sum_{\mathrm{i}} \gamma_{\mathrm{x}}^{\mathrm{i}} \mu_{\mathrm{i}}(\tilde{\boldsymbol{\rho}})\right) \tilde{\mathbf{w}}+\left(\sum_{\mathrm{i}} \gamma_{\mathrm{r}}^{\mathrm{i}} \mu_{\mathrm{i}}(\tilde{\boldsymbol{\rho}})\right) \dot{\mathbf{r}}
\end{aligned}
$$

where $\phi_{\mathrm{x}}^{\mathrm{i}}, \phi_{\mathrm{r}}^{\mathrm{i}}, \gamma_{\mathrm{x}}^{\mathrm{i}}$ and $\gamma_{\mathrm{r}}^{\mathrm{i}}$ are constant matrices. The corresponding local models, from which the blended multiple model system is constituted, are

$$
\begin{aligned}
& \dot{\overline{\mathbf{x}}}_{\mathrm{i}}=\overline{\mathbf{w}}_{\mathrm{i}} \\
& \dot{\overline{\mathbf{w}}}_{\mathrm{i}}=\phi_{\mathrm{x}}^{\mathrm{i}} \overline{\mathbf{w}}_{\mathrm{i}}+\phi_{\mathrm{r}}^{\mathrm{i}} \dot{\mathbf{r}} \\
& \dot{\overline{\mathbf{y}}}_{\mathrm{i}}=\gamma_{\mathrm{x}}^{\mathrm{i}} \overline{\mathbf{w}}_{\mathrm{i}}+\gamma_{\mathrm{r}}^{\mathrm{i}} \dot{\mathbf{r}}
\end{aligned}
$$

It should be noted that the local models are linear. Assume that the local models satisfy

$$
\phi_{x}^{\mathrm{i}}=\phi_{\mathbf{x}}\left(\mathbf{x}_{\mathrm{i}}, \mathbf{r}_{\mathrm{i}}\right), \quad \phi_{\mathbf{r}}^{\mathrm{i}}=\phi_{\mathbf{r}}\left(\mathbf{x}_{\mathrm{i}}, \mathbf{r}_{\mathrm{i}}\right), \gamma_{\mathbf{x}}^{\mathrm{i}}=\gamma_{\mathbf{x}}\left(\mathbf{x}_{\mathrm{i}}, \mathbf{r}_{\mathrm{i}}\right), \gamma_{\mathbf{r}}^{\mathrm{i}}=\gamma_{\mathbf{r}}\left(\mathbf{x}_{\mathrm{i}}, \mathbf{r}_{\mathrm{i}}\right)
$$

where

$$
\begin{aligned}
& \phi_{\mathbf{x}}\left(\mathbf{x}_{\mathrm{i}}, \mathbf{r}_{\mathrm{i}}\right)=\left.\nabla_{\mathbf{x}} \phi(\mathbf{x}, \mathbf{r})\right|_{\left(\mathbf{x}_{\mathrm{i}}, \mathbf{r}_{\mathrm{i}}\right)}, \quad \phi_{\mathbf{r}}\left(\mathbf{x}_{\mathrm{i}}, \mathbf{r}_{\mathrm{i}}\right)=\left.\nabla_{\mathbf{r}} \phi(\mathbf{x}, \mathbf{r})\right|_{\left(\mathbf{x}_{\mathrm{i}}, \mathbf{r}_{\mathrm{i}}\right)} \\
& \gamma_{\mathbf{x}}\left(\mathbf{x}_{\mathrm{i}}, \mathbf{r}_{\mathrm{i}}\right)=\left.\nabla_{\mathbf{x}} \gamma(\mathbf{x}, \mathbf{r})\right|_{\left(\mathbf{x}_{\mathrm{i}}, \mathbf{r}_{\mathrm{i}}\right)}, \gamma_{\mathbf{r}}\left(\mathbf{x}_{\mathrm{i}}, \mathbf{r}_{\mathrm{i}}\right)=\left.\nabla_{\mathbf{r}} \gamma(\mathbf{x}, \mathbf{r})\right|_{\left(\mathbf{x}_{\mathrm{i}}, \mathbf{r}_{\mathrm{i}}\right)}
\end{aligned}
$$

and $\phi(\bullet, \bullet)$ and $\gamma(\bullet, \bullet)$ are differentiable nonlinear functions with Lipschitz continuous first derivatives. Each local model then corresponds to the velocity-based linearisation, at the operating point $\left(\tilde{\mathbf{x}}_{\mathrm{i}}, \mathbf{r}_{\mathrm{i}}\right)$, of the nonlinear system

$$
\dot{\mathbf{x}}=\phi(\mathbf{x}, \mathbf{r}), \quad \mathbf{y}=\gamma(\mathbf{x}, \mathbf{r})
$$

Since the functions, $\phi(\bullet, \bullet)$ and $\gamma(\bullet, \bullet)$, are arbitrary (other than being required to be smooth), this requirement is simply a smoothness condition which serves a role similar to the regularisation measures required with blended multiple model systems, (1). In the extreme situation where an infinite number of local models are employed and the $\left(\tilde{\mathbf{x}}_{\mathrm{i}}, \mathbf{r}_{\mathrm{i}}\right)$ form a continuum in the operating space, this condition can be interpreted as an integrability condition: whilst always satisfied in the scalar case, it is a rather strong condition in the multi-dimensional case when $\mathrm{n}$ and/or $\mathrm{m}$ are greater than unity. However, in the situation considered in this paper, where the number of local models is finite and small, the condition is very weak since it only requires that the coefficients of the local models can be interpolated by integrable functions.

Suppose that the blended multiple model system is evolving along a trajectory in $\Phi$ and at time, $\mathrm{t}_{1}$, the trajectory has reached the point, $\left(\tilde{\mathbf{x}}_{1}, \mathbf{r}_{1}\right)$. Similarly to the discussion in section 3.1 concerning the relationship of (25)-(27) to (29)-(31), the solution to the frozen form of (37) at the operating point $\left(\tilde{\mathbf{x}}_{1}, \mathbf{r}_{1}\right)$

$$
\begin{aligned}
& \dot{\check{\mathbf{x}}}=\breve{\mathbf{w}} \\
& \dot{\check{\mathbf{w}}}=\left(\sum_{\mathrm{i}} \phi_{\mathrm{x}}^{\mathrm{i}} \mu_{\mathrm{i}}\left(\tilde{\rho}_{1}\right)\right) \breve{\mathbf{w}}+\left(\sum_{\mathrm{i}} \phi_{\mathbf{r}}^{\mathrm{i}} \mu_{\mathrm{i}}\left(\tilde{\rho}_{1}\right)\right) \dot{\mathbf{r}} \\
& \dot{\check{\mathbf{y}}}=\left(\sum_{\mathrm{i}} \gamma_{\mathrm{x}}^{\mathrm{i}} \mu_{\mathrm{i}}\left(\tilde{\rho}_{1}\right)\right) \breve{\mathbf{w}}+\left(\sum_{\mathrm{i}} \gamma_{\mathrm{r}}^{\mathrm{i}} \mu_{\mathrm{i}}\left(\tilde{\rho}_{1}\right)\right) \dot{\mathbf{r}} \\
& \tilde{\rho}_{\mathbf{1}}=\nabla_{\mathbf{x}} \tilde{\rho} \tilde{\mathbf{x}}_{\mathbf{1}}+\nabla_{\mathbf{r}} \tilde{\boldsymbol{\rho}} \mathbf{r}_{\mathbf{1}}
\end{aligned}
$$

with initial conditions 


$$
\breve{\mathbf{x}}\left(\mathrm{t}_{1}\right)=\tilde{\mathbf{x}}\left(\mathrm{t}_{1}\right), \quad \breve{\mathbf{w}}\left(\mathrm{t}_{1}\right)=\tilde{\mathbf{w}}\left(\mathrm{t}_{1}\right), \quad \breve{\mathbf{y}}\left(\mathrm{t}_{1}\right)=\tilde{\mathbf{y}}\left(\mathrm{t}_{1}\right)
$$

is, initially at time $t_{1}$, tangential to the solution to the blended multiple model system, (37). Indeed, $\breve{\mathbf{x}}$ is a second-order approximation to $\tilde{\mathbf{x}}, \breve{\mathbf{w}}$ is a first-order approximation to $\widetilde{\mathbf{w}}$ and $\breve{\mathbf{y}}$ is a first-order approximation to $\tilde{\mathbf{y}}$. Hence, the dynamics of the blended multiple model system, locally to the operating point $\left(\tilde{\mathbf{x}}_{1}, \mathbf{r}_{1}\right)$, are indicated by the dynamics of the corresponding frozen-form linear system, (42). Clearly, the frozen-form, (42), of the blended multiple model system is directly related to the local models, (38), from which it is constructed. Specifically, the frozen-form at the operating point, $\left(\tilde{\mathbf{x}}_{\mathbf{1}}, \mathbf{r}_{\mathbf{1}}\right)$, is a weighted linear combination of the local models with the weighting assigned to each local model just the value of the corresponding validity function at $\left(\widetilde{\mathbf{x}}_{\mathbf{1}}, \mathbf{r}_{1}\right)$. Hence, the dynamics of the blended multiple model system are directly related to the local models as required; that is, the dynamics are described by a straightforward blend of the local models.

The weighted linear combination of the solutions to the local models, (38), satisfies

$$
\begin{aligned}
& \sum_{\mathrm{i}} \dot{\overline{\mathbf{x}}}_{\mathrm{i}} \mu_{\mathrm{i}}\left(\tilde{\rho}_{1}\right)=\sum_{\mathrm{i}} \overline{\mathbf{w}}_{\mathrm{i}} \mu_{\mathrm{i}}\left(\tilde{\rho}_{1}\right) \\
& \sum_{\mathrm{i}} \dot{\overline{\mathbf{w}}}_{\mathrm{i}} \mu_{\mathrm{i}}\left(\tilde{\rho}_{1}\right)=\left(\sum_{\mathrm{i}} \phi_{\mathrm{x}}^{\mathrm{i}} \mu_{\mathrm{i}}\left(\tilde{\rho}_{1}\right)\right)\left(\sum_{\mathrm{i}} \overline{\mathbf{w}}_{\mathrm{i}} \mu_{\mathrm{i}}\left(\tilde{\rho}_{1}\right)\right)+\left(\sum_{\mathrm{i}} \phi_{\mathrm{r}}^{\mathrm{i}} \mu_{\mathrm{i}}\left(\tilde{\rho}_{1}\right)\right) \dot{\mathbf{r}}+\left(\sum_{\mathrm{i}} \phi_{\mathrm{x}}^{\mathrm{i}} \overline{\mathbf{w}}_{\mathrm{i}} \mu_{\mathrm{i}}\left(\tilde{\rho}_{1}\right)-\sum_{\mathrm{i}} \sum_{\mathrm{j}} \phi_{\mathrm{x}}^{\mathrm{i}} \overline{\mathbf{w}}_{\mathrm{j}} \mu_{\mathrm{i}}\left(\tilde{\rho}_{1}\right) \mu_{\mathrm{j}}\left(\tilde{\rho}_{1}\right)\right)(44) \\
& \sum_{\mathrm{i}} \dot{\bar{y}}_{\mathrm{i}} \mu_{\mathrm{i}}\left(\tilde{\boldsymbol{\rho}}_{1}\right)=\left(\sum_{\mathrm{i}} \gamma_{\mathrm{x}}^{\mathrm{i}} \mu_{\mathrm{i}}\left(\tilde{\rho}_{1}\right)\right)\left(\sum_{\mathrm{i}} \overline{\mathbf{w}}_{\mathrm{i}} \mu_{\mathrm{i}}\left(\tilde{\rho}_{1}\right)\right)+\left(\sum_{\mathrm{i}} \gamma_{\mathrm{r}}^{\mathrm{i}} \mu_{\mathrm{i}}\left(\tilde{\rho}_{1}\right)\right) \dot{\mathbf{r}}+\left(\sum_{\mathrm{i}} \gamma_{\mathrm{x}}^{\mathrm{i}} \overline{\mathbf{w}}_{\mathrm{i}} \mu_{\mathrm{i}}\left(\tilde{\boldsymbol{\rho}}_{1}\right)-\sum_{\mathrm{i}} \sum_{\mathrm{j}} \gamma_{\mathrm{x}}^{\mathrm{i}} \overline{\mathbf{w}}_{\mathrm{j}} \mu_{\mathrm{i}}\left(\tilde{\rho}_{1}\right) \mu_{\mathrm{j}}\left(\tilde{\boldsymbol{\rho}}_{1}\right)\right)
\end{aligned}
$$

which may be reformulated as

$$
\begin{aligned}
& \dot{\breve{\mathbf{x}}}^{*}=\breve{\mathbf{w}}^{*} \\
& \dot{\breve{\mathbf{w}}}^{*}=\left(\sum_{\mathrm{i}} \phi_{\mathrm{x}}^{\mathrm{i}} \mu_{\mathrm{i}}\left(\tilde{\rho}_{1}\right)\right) \breve{\mathbf{w}}^{*}+\left(\sum_{\mathrm{i}} \phi_{\mathrm{r}}^{\mathrm{i}} \mu_{\mathrm{i}}\left(\tilde{\rho}_{1}\right)\right) \dot{\mathbf{r}}+\varepsilon_{\mathbf{w}^{*}} \\
& \dot{\overrightarrow{\mathbf{y}}}^{*}=\left(\sum_{\mathrm{i}} \gamma_{\mathrm{x}}^{\mathrm{i}} \mu_{\mathrm{i}}\left(\tilde{\rho}_{1}\right)\right) \breve{\mathbf{w}}^{*}+\left(\sum_{\mathrm{i}} \gamma_{\mathrm{r}}^{\mathrm{i}} \mu_{\mathrm{i}}\left(\tilde{\rho}_{1}\right)\right) \dot{\mathbf{r}}+\varepsilon_{\mathbf{y}^{*}}
\end{aligned}
$$

where

$$
\begin{array}{ll}
\breve{\mathbf{x}}^{*}=\sum_{\mathrm{i}} \overline{\mathbf{x}}_{\mathrm{i}} \mu_{\mathrm{i}}\left(\tilde{\boldsymbol{\rho}}_{1}\right), \breve{\mathbf{w}}^{*}=\left(\sum_{\mathrm{i}} \overline{\mathbf{w}}_{\mathrm{i}} \mu_{\mathrm{i}}\left(\tilde{\boldsymbol{\rho}}_{1}\right)\right), \quad \breve{\mathbf{y}}^{*}=\sum_{\mathrm{i}} \dot{\overline{\mathbf{y}}}_{\mathrm{i}} \mu_{\mathrm{i}}\left(\tilde{\rho}_{1}\right) \\
\varepsilon_{\mathbf{w}^{*}}=\left(\sum_{\mathrm{i}} \sum_{\mathrm{j}}\left(\phi_{\mathrm{x}}^{\mathrm{i}}-\phi_{\mathrm{x}}^{\mathrm{j}}\right) \overline{\mathbf{w}}_{\mathrm{i}} \mu_{\mathrm{j}}\left(\tilde{\rho}_{1}\right) \mu_{\mathrm{i}}\left(\tilde{\rho}_{1}\right)\right), \quad \varepsilon_{\mathbf{y}^{*}}=\left(\sum_{\mathrm{i}} \sum_{\mathrm{j}}\left(\gamma_{\mathrm{x}}^{\mathrm{i}}-\gamma_{\mathrm{x}}^{\mathrm{j}}\right) \overline{\mathbf{w}}_{\mathrm{i}} \mu_{\mathrm{i}}\left(\tilde{\rho}_{1}\right) \mu_{\mathrm{j}}\left(\tilde{\rho}_{1}\right)\right)
\end{array}
$$

It follows that, provided $\varepsilon_{w^{*}}$ and $\varepsilon_{\mathbf{y}^{*}}$ are sufficiently small, the solution to the frozen form is described by the weighted linear combination of the solutions to the local models. Since $\phi_{\mathbf{x}}(\tilde{\mathbf{x}}, \mathbf{r})$ and $\gamma_{\mathbf{x}}(\tilde{\mathbf{x}}, \mathbf{r})$ are Lipschitz continuous in $\hat{\Phi}$, there exists a positive finite constant, L, such that

$$
\begin{aligned}
& \left|\varepsilon_{\mathbf{w}^{*}}\right| \leq\left(\sum_{\mathrm{i}} \sum_{\mathrm{j}} \mathrm{L}\left(\left|\tilde{\mathbf{x}}_{\mathrm{i}}-\tilde{\mathbf{x}}_{\mathrm{j}}\right|+\left|\mathbf{r}_{\mathrm{i}}-\mathbf{r}_{\mathrm{j}}\right|\right)\left|\overline{\mathbf{w}}_{\mathrm{i}}\right| \mu_{\mathrm{j}}\left(\tilde{\rho}_{1}\right) \mu_{\mathrm{i}}\left(\tilde{\rho}_{1}\right)\right) \\
& \left|\varepsilon_{\mathbf{y}^{*}}\right| \leq\left(\sum_{\mathrm{i}} \sum_{\mathrm{j}} \mathrm{L}\left(\left|\tilde{\mathbf{x}}_{\mathrm{i}}-\tilde{\mathbf{x}}_{\mathrm{j}}\right|+\left|\mathbf{r}_{\mathrm{i}}-\mathbf{r}_{\mathrm{j}}\right|\right)\left|\overline{\mathbf{w}}_{\mathrm{i}}\right| \mu_{\mathrm{j}}\left(\tilde{\rho}_{1}\right) \mu_{\mathrm{i}}\left(\tilde{\rho}_{1}\right)\right)
\end{aligned}
$$

Hence, the magnitudes of $\varepsilon_{\mathbf{w}^{*}}$ and $\varepsilon_{\mathbf{y}^{*}}$ are proportional to the distance between the centres, $\left(\tilde{\mathbf{x}}_{\mathrm{i}}, \mathbf{r}_{\mathrm{i}}\right)$, of the local models and the overlap in the validity functions, $\mu_{\mathrm{i}}$. Evidently, $\varepsilon_{\mathbf{w}^{*}}$ and $\varepsilon_{\mathbf{y}^{*}}$ tend to zero as the distance between the centres tends to zero provided the overlap is confined to neighbouring local models and the solutions, $\overline{\mathbf{w}}_{\mathrm{i}}$, are bounded. When the magnitudes of $\varepsilon_{\mathbf{w}^{*}}$ and $\varepsilon_{\mathbf{y}^{*}}$ are sufficiently small, they can be neglected in (45). In these circumstances, the solution to (42) (and therefore the solution, locally to $\left(\tilde{\mathbf{x}}_{1}, \mathbf{r}_{1}\right)$, to the blended multiple model system) can be approximated by the weighted linear combination of the solutions to the local models. Of course, the degree of accuracy to which this approximation corresponds, like the degree of accuracy of representation of the blended multiple model system, is related to the number of local models and a suitable, contextually dependent, compromise must be reached to keep this number small. 
Furthermore, consider the nonlinear system, (14), for which the velocity-based linearisation family is (25)-(27) and let

$$
\begin{array}{ll}
\phi_{x}^{\mathrm{i}}=\mathbf{A}+\nabla_{\mathrm{x}} \mathbf{f}\left(\rho\left(\tilde{\mathbf{x}}_{\mathrm{i}}, \mathbf{r}_{\mathrm{i}}\right)\right) \nabla_{\mathrm{x}} \rho, & \phi_{\mathrm{r}}^{\mathrm{i}}=\mathbf{B}+\nabla_{\mathbf{r}} \mathbf{f}\left(\rho\left(\tilde{\mathbf{x}}_{\mathrm{i}}, \mathbf{r}_{\mathrm{i}}\right)\right) \nabla_{\mathrm{r}} \rho \\
\gamma_{\mathrm{x}}^{\mathrm{i}}=\mathbf{C}+\nabla_{\mathrm{x}} \mathbf{g}\left(\rho\left(\tilde{\mathbf{x}}_{\mathrm{i}}, \mathbf{r}_{\mathrm{i}}\right)\right) \nabla_{\mathrm{x}} \rho, & \gamma_{\mathrm{r}}^{\mathrm{i}}=\mathbf{D}+\nabla_{\mathrm{x}} \mathbf{g}\left(\rho\left(\tilde{\mathbf{x}}_{\mathrm{i}}, \mathbf{r}_{\mathrm{i}}\right)\right) \nabla_{\mathrm{r}} \rho \\
\nabla_{\mathrm{x}} \tilde{\rho}=\nabla_{\mathrm{x}} \rho, \quad \nabla_{\mathbf{r}} \tilde{\rho}=\nabla_{\mathbf{r}} \rho &
\end{array}
$$

When $\mu_{\mathrm{i}}\left(\tilde{\mathbf{x}}_{\mathrm{i}}, \mathbf{r}_{\mathrm{i}}\right)$ is unity and $\mu_{\mathrm{j}}\left(\tilde{\mathbf{x}}_{\mathrm{i}}, \mathbf{r}_{\mathrm{i}}\right), \mathrm{j} \neq \mathrm{i}$, is zero, the frozen-form of the blended multiple model system at the operating point $\left(\tilde{\mathbf{x}}_{\mathrm{i}}, \mathbf{r}_{\mathrm{i}}\right)$ is precisely the velocity-based linearisation of (14) at $\left(\tilde{\mathbf{x}}_{\mathrm{i}}, \mathbf{r}_{\mathrm{i}}\right)$. At other operating points, $\left(\tilde{\mathbf{x}}_{1}, \mathbf{r}_{1}\right)$, the frozen form, (42), is the approximation to the velocity-based linearisation of (14) obtained by interpolating, using the validity functions, between the velocity-based linearisations at the operating points, $\left(\tilde{\mathbf{x}}_{\mathrm{i}}, \mathbf{r}_{\mathrm{i}}\right)$. A frozen form, (42), is associated with every operating point and the resulting family of frozen forms is an approximation to the velocity-based linearisation family of (14). Hence, the blended multiple model system, (37), is an approximation to the velocity-form, (29)-(31), of the nonlinear system, (14), and the solution $\tilde{\mathbf{x}}$ to the blended multiple model system approximates the solution $\mathbf{x}$ to (14) over any finite time interval (see, for example, Khalil 1992 Theorem 2.5). Indeed, locally to the operating point, $\left(\widetilde{\mathbf{x}}_{1}, \mathbf{r}_{1}\right)$, the solution $\mathbf{x}$ to the nonlinear system, (14), is approximated by the solution to the velocitybased linearisation, (25)-(27), which in turn is approximated by the solution $\overrightarrow{\mathbf{x}}$ to the approximate velocitybased linearisation, (42).

\subsection{Higher-order local models}

In section 3.2, it is assumed that $\phi_{\mathrm{x}}^{\mathrm{i}}, \phi_{\mathrm{r}}^{\mathrm{i}}, \gamma_{\mathrm{x}}^{\mathrm{i}}$ and $\gamma_{\mathrm{r}}^{\mathrm{i}}$ are constant in the local models, (38). However, the analysis is essentially unchanged when $\phi_{\mathbf{x}}^{\mathrm{i}}, \phi_{\mathbf{r}}^{\mathrm{i}}, \boldsymbol{\gamma}_{\mathbf{x}}^{\mathrm{i}}$ and $\boldsymbol{\gamma}_{\mathbf{r}}^{\mathrm{i}}$ are allowed to vary with $\tilde{\mathbf{x}}$ and $\mathbf{r}$. For example, when the local models are

$$
\begin{aligned}
& \dot{\overline{\mathbf{x}}}=\overline{\mathbf{w}} \\
& \dot{\overline{\mathbf{w}}}=\phi_{\mathbf{x}}^{\mathrm{i}}(\tilde{\mathbf{x}}, \mathbf{r}) \overline{\mathbf{w}}+\phi_{\mathbf{r}}^{\mathrm{i}}(\tilde{\mathbf{x}}, \mathbf{r}) \dot{\mathbf{r}} \\
& \dot{\overline{\mathbf{y}}}=\gamma_{\mathbf{x}}^{\mathrm{i}}(\tilde{\mathbf{x}}, \mathbf{r}) \overline{\mathbf{w}}+\gamma_{\mathbf{r}}^{\mathrm{i}}(\tilde{\mathbf{x}}, \mathbf{r}) \dot{\mathbf{r}}
\end{aligned}
$$

where

$$
\begin{array}{ll}
\phi_{x}^{\mathrm{i}}\left(\tilde{\mathbf{x}}_{\mathrm{i}}, \mathbf{r}_{\mathrm{i}}\right)=\mathbf{A}+\nabla_{\mathrm{x}} \mathbf{f}\left(\rho\left(\tilde{\mathbf{x}}_{\mathrm{i}}, \mathbf{r}_{\mathrm{i}}\right)\right) \nabla_{\mathrm{x}} \rho, & \phi_{\mathbf{r}}^{\mathrm{i}}\left(\tilde{\mathbf{x}}_{\mathrm{i}}, \mathbf{r}_{\mathrm{i}}\right)=\mathbf{B}+\nabla_{\mathbf{r}} \mathbf{f}\left(\rho\left(\tilde{\mathbf{x}}_{\mathrm{i}}, \mathbf{r}_{\mathrm{i}}\right)\right) \nabla_{\mathrm{r}} \rho \\
\gamma_{\mathrm{x}}^{\mathrm{i}}\left(\tilde{\mathbf{x}}_{\mathrm{i}}, \mathbf{r}_{\mathrm{i}}\right)=\mathbf{C}+\nabla_{\mathrm{x}} \mathbf{g}\left(\rho\left(\tilde{\mathbf{x}}_{\mathrm{i}}, \mathbf{r}_{\mathrm{i}}\right)\right) \nabla_{\mathrm{x}} \rho, & \gamma_{\mathrm{r}}^{\mathrm{i}}\left(\tilde{\mathbf{x}}_{\mathrm{i}}, \mathbf{r}_{\mathrm{i}}\right)=\mathbf{D}+\nabla_{\mathrm{x}} \mathbf{g}\left(\rho\left(\tilde{\mathbf{x}}_{\mathrm{i}}, \mathbf{r}_{\mathrm{i}}\right)\right) \nabla_{\mathrm{r}} \rho \\
\nabla_{\mathrm{x}} \tilde{\rho}=\nabla_{\mathrm{x}} \rho, \quad \nabla_{\mathbf{r}} \tilde{\rho}=\nabla_{\mathrm{r}} \rho &
\end{array}
$$

each local model approximates the velocity-based linearisations of the nonlinear system, (14), in a neighbourhood about the operating point, $\left(\tilde{\mathbf{x}}_{\mathrm{i}}, \mathbf{r}_{\mathrm{i}}\right)$, thereby augmenting the interpolation provided by the validity functions. Of course, the associated potential improvement in accuracy must be balanced against the increased complexity of the local models.

\subsection{Velocity-based blended multiple controllers}

The requirement for a direct relationship between the dynamics of a blended multiple model system and the local models is motivated, in part, by control design requirements. For example, in the context of local model networks, Hunt \& Johansen (1997) consider the design of a nonlinear controller whereby a linear controller is designed for each local model and the members of the family of linear controllers obtained are blended to obtain a nonlinear controller. However, the design procedure is complicated considerably by the lack of a direct relationship between the dynamics of such blended multiple model systems and the local models on which the control design is based. Moreover, the local models employed are affine and do not provide continuity with established linear methods. In particular, they do not possess the superposition property which is fundamental to linear systems. The magnitude of the inhomogeneous term in an affine local model may be extremely large and can strongly influence the solution. Furthermore, when the inhomogeneous term varies from one local model to another, it introduces an implicit feedback from the state and/or input of the overall blended multiple model system. Hence, the inhomogeneous term cannot, in general, simply be treated as a small disturbance to be rejected by the controller and this leads to Hunt $\&$ 
Johansen (1997) introducing slow variation requirements which are quite distinct from those encountered in conventional gain-scheduling.

In contrast to conventional blended multiple model systems, the dynamics of the velocity-based blended multiple model system, (37), in the blended regions are directly related to the local models. Moreover, the local models are linear. Control design based on a blended multiple model plant representation is thereby considerably facilitated. Consider the nonlinear plant, (37), and the nonlinear controller

$$
\begin{aligned}
& \dot{\tilde{\mathbf{x}}}_{\mathrm{c}}=\tilde{\mathbf{w}}_{\mathrm{c}} \\
& \dot{\tilde{\mathbf{w}}}_{\mathrm{c}}=\left(\sum_{\mathrm{i}} \phi_{\mathrm{x}_{\mathrm{c}}}^{\mathrm{i}} \mu_{\mathrm{i}}(\tilde{\boldsymbol{\rho}})\right) \tilde{\mathbf{w}}_{\mathrm{c}}+\left(\sum_{\mathrm{i}} \phi_{\mathrm{r}_{\mathrm{c}}}^{\mathrm{i}} \mu_{\mathrm{i}}(\tilde{\boldsymbol{\rho}})\right) \dot{\mathbf{r}}_{\mathrm{c}} \\
& \dot{\tilde{\mathbf{y}}}_{\mathrm{c}}=\left(\sum_{\mathrm{i}} \gamma_{\mathrm{x}_{\mathrm{c}}}^{\mathrm{i}} \mu_{\mathrm{i}}(\tilde{\boldsymbol{\rho}})\right) \tilde{\mathbf{w}}_{\mathrm{c}}+\left(\sum_{\mathrm{i}} \gamma_{\mathrm{r}_{\mathrm{c}}}^{\mathrm{i}} \mu_{\mathrm{i}}(\tilde{\boldsymbol{\rho}})\right) \dot{\mathbf{r}}_{\mathrm{c}}
\end{aligned}
$$

where $\tilde{\mathbf{r}}_{\mathbf{c}} \in \mathfrak{R}^{\mathbf{m}_{c}}$ denotes the input to the controller, $\tilde{\mathbf{y}}_{\mathbf{c}} \in \mathfrak{R}^{p_{c}}$ the output and $\tilde{\mathbf{x}}_{\mathbf{c}} \in \mathfrak{R}^{n_{c}}$ the state. Since the requirement is to design a feedback controller, to facilitate the analysis it is assumed the input, $\tilde{\mathbf{r}}$, to the plant is the output, $\tilde{\mathbf{y}}_{\mathbf{c}}$, of the controller and the input vector, $\tilde{\mathbf{r}}_{\mathbf{c}}$, to the controller is the error, $\tilde{\mathbf{y}}_{\text {ref }}-\tilde{\mathbf{y}}_{\text {. }}$ Assume, in addition, that $\gamma_{\mathrm{r}}^{\mathrm{i}}$ and $\gamma_{\mathbf{r}_{\mathrm{c}}}^{\mathrm{i}}$ are zero. These assumptions are employed only to simplify the analysis and it is straightforward to extend to analysis to include more general configurations, including two degree of freedom controllers and situations where $\gamma_{\mathbf{r}}^{i}$ and $\gamma_{\mathbf{r}_{\mathbf{c}}}^{\mathrm{i}}$ are not zero. The combined closed-loop dynamics are

$$
\begin{aligned}
& \dot{\tilde{\mathbf{x}}}=\tilde{\mathbf{w}}, \quad \dot{\tilde{\mathbf{x}}}_{\mathrm{c}}=\tilde{\mathbf{w}}_{\mathrm{c}}
\end{aligned}
$$

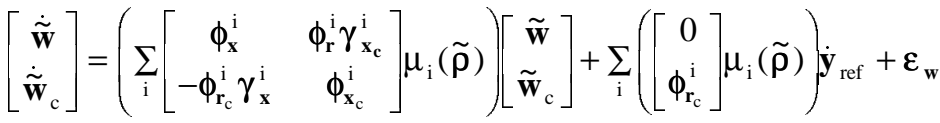

$$
\begin{aligned}
& \dot{\tilde{\mathbf{y}}}=\left(\sum_{\mathrm{i}}\left[\begin{array}{ll}
\gamma_{\mathrm{x}}^{\mathrm{i}} & 0
\end{array}\right] \mu_{\mathrm{i}}(\tilde{\boldsymbol{\rho}})\right)\left[\begin{array}{c}
\tilde{\mathbf{w}} \\
\tilde{\mathbf{w}}_{\mathrm{c}}
\end{array}\right]
\end{aligned}
$$

where

$$
\varepsilon_{\mathrm{w}}=\sum_{\mathrm{i}} \sum_{\mathrm{j}}\left[\begin{array}{l}
\phi_{\mathrm{r}}^{\mathrm{i}}\left(\gamma_{\mathrm{x}_{\mathrm{c}}}^{\mathrm{j}}-\gamma_{\mathrm{x}_{\mathrm{c}}}^{\mathrm{i}}\right) \tilde{\mathbf{w}}_{\mathrm{c}} \\
-\phi_{\mathbf{r}_{c}}^{\mathrm{i}}\left(\gamma_{\mathrm{x}}^{\mathrm{j}}-\gamma_{\mathrm{x}}^{\mathrm{i}}\right) \tilde{\mathbf{w}}
\end{array}\right] \mu_{\mathrm{i}}(\tilde{\boldsymbol{\rho}}) \mu_{\mathrm{j}}(\tilde{\rho})
$$

The perturbation term, $\varepsilon_{\mathrm{w}}$, vanishes when

$$
\left(\sum_{i} \gamma_{x}^{i} \mu_{i}(\tilde{\rho})\right)=\mathbf{C},\left(\sum_{i} \gamma_{x_{c}}^{i} \mu_{i}(\tilde{\rho})\right)=\mathbf{C}_{\mathbf{c}}
$$

with $\mathbf{C}$ and $\mathbf{C}_{\mathbf{c}}$ constant matrices. This requires the output of the plant and the output of the controller, respectively, to be linearly related to the state of the plant and the state of the controller. More generally, the magnitude of $\varepsilon_{\mathrm{w}}$ is dependent on the distance between the centres, $\left(\tilde{\mathbf{x}}_{\mathrm{i}}, \mathbf{r}_{\mathrm{i}}\right)$, of the local models and the overlap in the validity functions, $\mu_{\mathrm{i}}$ (similarly to $\varepsilon_{\mathbf{w}^{*}}$ and $\varepsilon_{\mathbf{y}^{*}}$ ). Clearly, the discussion following (48)-(49) is section 3.2 also applies to $\varepsilon_{\mathrm{w}}$ and, when required, the magnitude of $\varepsilon_{\mathrm{w}}$ can be kept sufficiently small that it can be neglected.

Observe that the dynamics of the closed-loop combination of the $\mathrm{i}^{\text {th }}$ individual plant local model and the $\mathrm{i}^{\text {th }}$ individual controller local model are

$$
\begin{aligned}
& \dot{\overline{\mathbf{x}}}_{\mathrm{i}}=\overline{\mathbf{w}}_{\mathrm{i}}, \quad \dot{\overline{\mathbf{x}}}_{\mathrm{c}_{\mathrm{i}}}=\overline{\mathbf{w}}_{\mathrm{c}_{\mathrm{i}}} \\
& {\left[\begin{array}{c}
\dot{\mathbf{w}}_{\mathrm{i}} \\
\dot{\overline{\mathbf{w}}}_{\mathrm{c}_{\mathrm{i}}}
\end{array}\right]=\left[\begin{array}{cc}
\phi_{\mathrm{x}}^{\mathrm{i}} & \phi_{\mathrm{r}}^{\mathrm{i}} \gamma_{\mathbf{x}_{c}}^{\mathrm{i}} \\
-\phi_{\mathbf{r}_{\mathrm{c}}}^{\mathrm{i}} \gamma_{\mathrm{x}}^{\mathrm{i}} & \phi_{\mathbf{x}_{\mathrm{c}}}^{\mathrm{i}}
\end{array}\right]\left[\begin{array}{c}
\overline{\mathbf{w}}_{\mathrm{i}} \\
\overline{\mathbf{w}}_{\mathrm{c}_{\mathrm{i}}}
\end{array}\right]+\left[\begin{array}{c}
0 \\
\phi_{\mathrm{r}_{\mathrm{c}}}^{\mathrm{i}}
\end{array} \dot{\mathbf{y}}_{\mathrm{ref}}\right.} \\
& \dot{\overline{\mathbf{y}}}_{\mathrm{i}}=\left[\begin{array}{ll}
\gamma_{\mathrm{x}}^{\mathrm{i}} & \gamma_{\mathrm{r}}^{\mathrm{i}} \gamma_{\mathbf{x}_{c}}^{\mathrm{i}}
\end{array}\right]\left[\begin{array}{c}
\overline{\mathbf{w}}_{\mathrm{i}} \\
\overline{\mathbf{w}}_{\mathrm{c}_{\mathrm{i}}}
\end{array}\right]
\end{aligned}
$$

It is evident that, provided the perturbation term, $\varepsilon_{\mathrm{w}}$, is sufficiently small, the closed-loop dynamics, (54), of the blended multiple model system are described simply by the weighted combination of the closed-loop 
local models. Owing to this very direct relationship, and the linearity of the local models, the velocitybased blended multiple model approach clearly offers the potential to facilitate controller design.

\subsection{Realisation of velocity-based blended multiple model systems}

Owing to the differentiation and integration operations associated with the velocity-form local models, the order of the velocity-based blended multiple model system, (42), is greater than the order of (1). Moreover, some care is required when realising the blended multiple model system, (42), since the input is $\dot{\mathbf{r}}$ rather than $\mathbf{r}$.

Regarding the order of (42), it should be noted that the quantity, $\tilde{\mathbf{x}}$, only affects the input-output characteristics of (42) through its influence on $\tilde{\rho}(\tilde{\mathbf{x}}, \mathbf{r})$, and thereby on $\mu_{\mathrm{i}}$. Hence, (42) can be reformulated equivalently as

$$
\begin{aligned}
& \dot{\tilde{\rho}}=\nabla_{x} \tilde{\rho} \tilde{\mathbf{w}}+\nabla_{r} \tilde{\rho} \dot{\mathbf{r}} \\
& \dot{\tilde{\mathbf{w}}}=\left(\sum_{i} \phi_{x}^{\mathrm{i}} \mu_{\mathrm{i}}(\tilde{\rho})\right) \tilde{\mathbf{w}}+\left(\sum_{\mathrm{i}} \phi_{\mathrm{r}}^{\mathrm{i}} \mu_{\mathrm{i}}(\tilde{\rho})\right) \dot{\mathbf{r}} \\
& \dot{\mathbf{y}}=\left(\sum_{\mathrm{i}} \gamma_{\mathrm{x}}^{\mathrm{i}} \mu_{\mathrm{i}}(\tilde{\rho})\right) \tilde{\mathbf{w}}+\left(\sum_{\mathrm{i}} \gamma_{\mathrm{r}}^{\mathrm{i}} \mu_{\mathrm{i}}(\tilde{\rho})\right) \dot{\mathbf{r}}
\end{aligned}
$$

Since the degree of $\tilde{\rho}$ is often lower than that of $\tilde{\mathbf{x}}$, the order of the formulation, (58), is frequently lower than that of (42). In the context of system identification, the initial condition of $\tilde{\rho}$ in (58) can be chosen arbitrarily since any constant offset is absorbed within the nonlinear validity functions, $\mu_{\mathrm{i}}$. When the mapping from $(\widetilde{\mathbf{x}}, \mathbf{r})$ is invertible in the sense that $\widetilde{\mathbf{x}}$ may be expressed as a function of $\widetilde{\mathbf{w}}, \mathbf{r}$ and $\tilde{\mathbf{y}}$, then $\tilde{\rho}$ is related algebraically to $(\tilde{\mathbf{w}}, \mathbf{r}, \tilde{\mathbf{y}})$ and the order of the blended multiple model system can be further reduced.

Regarding the input being $\dot{\mathbf{r}}$ rather than $\mathbf{r}$ in the velocity-based form, when the blended multiple model satisfies an integrability condition it may, by integrating, be reformulated as a nonlinear system of the form, (14) (Leith \& Leithead 1996, 1998b; see also the example in section 4 below). The input to the reformulated system is $\mathbf{r}$ but the terms in the resulting integrated system are related to the terms in the blended multiple model system by an integral equation. Alternatively, when the local models contain integral action, the blended multiple model system can be realised in velocity-based form (Leith \& Leithead 1996, 1998b). This has the distinct advantage that the elements in the realisation are related directly to (42). In the context of control systems, pure integral action is frequently encountered. In the context of system identification and modelling, the plant under consideration can be readily augmented with integral action. For example, system identification and modelling require the design and application of a suitable test input to provide information regarding the plant dynamics; rather than designing a test input corresponding to $\mathbf{r}$, it is straightforward to design instead a test input corresponding to $\dot{\mathbf{r}}$ which is then integrated to obtain the test input applied to the actual plant. Alternatively, when a control system associated with the plant contains integral action, this can be included with the plant dynamics for modelling purposes. The realisation of velocity-based systems, including situations where integral action is not present, is discussed in detail in Leith \& Leithead $(1996,1998$ b)

\section{Example}

Consider the second-order nonlinear system

$$
\left[\begin{array}{l}
\dot{x}_{1} \\
\dot{x}_{2}
\end{array}\right]=\left[\begin{array}{cc}
0 & 1 \\
0 & -Q
\end{array}\right]\left[\begin{array}{l}
\mathrm{x}_{1} \\
\mathrm{x}_{2}
\end{array}\right]+\left[\begin{array}{l}
0 \\
\mathrm{~b}
\end{array}\right] \mathrm{r}+\left[\begin{array}{c}
0 \\
-\mathrm{Q} \sin \left(\mathrm{x}_{1}\right)
\end{array}\right], \mathrm{y}=\left[\begin{array}{ll}
1 & 0
\end{array}\right]\left[\begin{array}{l}
\mathrm{x}_{1} \\
\mathrm{x}_{2}
\end{array}\right]
$$

where $\mathrm{Q}=29.46, \mathrm{~b}=1.21$. The nonlinearity is dependent on $\mathrm{x}_{1}$ and consideration is confined to the operating space where $\mathrm{x}_{1} \in[0, \pi]$. The first-order series expansion of (59) about the operating point $\left(\mathrm{x}_{10}, \mathrm{x}_{2 \mathrm{o}}, \mathrm{r}_{\mathrm{o}}\right)$ is 


$$
\left[\begin{array}{c}
\dot{\mathrm{x}}_{1} \\
\dot{\mathrm{x}}_{2}
\end{array}\right]=\left[\begin{array}{c}
0 \\
-\mathrm{Q} \sin \left(\mathrm{x}_{10}\right)+\mathrm{Q} \cos \left(\mathrm{x}_{10}\right) \mathrm{x}_{10}
\end{array}\right]+\left[\begin{array}{cc}
0 & 1 \\
-\mathrm{Q} \cos \left(\mathrm{x}_{10}\right) & -\mathrm{Q}
\end{array}\right]\left[\begin{array}{l}
\mathrm{x}_{1} \\
\overline{\mathrm{x}}_{2}
\end{array}\right]+\left[\begin{array}{l}
0 \\
\mathrm{~b}
\end{array}\right] \mathrm{r}, \quad \breve{\mathrm{y}}=\left[\begin{array}{ll}
1 & 0
\end{array}\right]\left[\begin{array}{l}
\mathrm{x}_{1} \\
\overline{\mathrm{x}}_{2}
\end{array}\right]
$$

A blended multiple model system with affine local models, obtained by directly blending the first-order series expansions about the operating points at which $\mathrm{x}_{1}$ is $0, \pi / 2$ and $\pi$, is

$$
\left[\begin{array}{c}
\tilde{\mathrm{x}}_{1} \\
\tilde{\mathrm{x}}_{2}
\end{array}\right]=\sum_{\mathrm{i}=1}^{3}\left(\alpha_{\mathrm{i}}+\mathbf{A}_{\mathrm{i}}\left[\begin{array}{c}
\tilde{\mathrm{x}}_{1} \\
\tilde{\mathrm{x}}_{2}
\end{array}\right]+\mathbf{B}_{\mathrm{i}} \mathrm{r}\right) \mu_{\mathrm{i}}\left(\tilde{\mathrm{x}}_{1}\right), \tilde{\mathrm{y}}=\sum_{\mathrm{i}=1}^{3} \mathbf{C}_{\mathrm{i}}\left[\begin{array}{l}
\tilde{\mathrm{x}}_{1} \\
\tilde{\mathrm{x}}_{2}
\end{array}\right] \mu_{\mathrm{i}}\left(\tilde{\mathrm{x}}_{1}\right)
$$

where

$$
\alpha_{\mathrm{i}}=\left[\begin{array}{c}
0 \\
-\mathrm{Q} \sin \left(\mathrm{x}_{1 \mathrm{i}}\right)+\mathrm{Q} \cos \left(\mathrm{x}_{1 \mathrm{i}}\right) \mathrm{x}_{1 \mathrm{i}}
\end{array}\right], \quad \mathbf{A}_{\mathrm{i}}=\left[\begin{array}{cc}
0 & 1 \\
-\mathrm{Q} \cos \left(\mathrm{x}_{1 \mathrm{i}}\right) & -\mathrm{Q}
\end{array}\right], \quad \mathbf{B}_{\mathrm{i}}=\left[\begin{array}{l}
0 \\
\mathrm{~b}
\end{array}\right] \mathrm{r}, \quad \mathbf{C}_{\mathrm{i}}=\left[\begin{array}{ll}
1 & 0
\end{array}\right]
$$

$\mathrm{x}_{11}=0, \mathrm{x}_{12}=\pi / 2, \mathrm{x}_{13}=\pi$ and the $\mu_{\mathrm{i}}$ are as depicted in figure 1 . In figure $2 \mathrm{a}$, the right-hand side of the differential equation for $\mathrm{x}_{2}$ in (59) is compared with the right-hand side of the differential equation for $\tilde{\mathrm{x}}_{2}$ in the blended multiple model system, (61) (with $\mathrm{x}_{2}$ and $\mathrm{r}$ zero). It can be seen that, owing to the blending of the inhomogeneous terms in the affine local models, an offset exists in the blended multiple model system compared to the nonlinear system, (59). The offset causes a considerable difference in the output of the blended multiple model system when compared to the output of the nonlinear system, (59). At the cost of weakening the connection between the local models and the first-order series expansions, (60), this offset is reduced by adjusting the inhomogeneous terms in the local models to

$$
\alpha_{\mathrm{i}}=\left[\begin{array}{c}
0 \\
-\mathrm{Q} \sin \left(\mathrm{x}_{1 \mathrm{i}}\right)+\mathrm{Q} \cos \left(\mathrm{x}_{1 \mathrm{i}}\right) \mathrm{x}_{1 \mathrm{i}}+3.0
\end{array}\right]
$$

It can be seen from figure $2 \mathrm{~b}$ that the right-hand side of the differential equation for $\tilde{\mathrm{x}}_{2}$ in the modified blended multiple model system is a somewhat better approximation to right-hand side of the $\mathrm{x}_{2}$ equation in (59). The output of the nonlinear system, (59), in response to a test input is depicted in figure 3a. The test input is quite demanding since it is selected so that the nonlinear system operates near the mid-point between two local models, where $x_{1}$ is approximately $\pi / 4$. The difference between the output of (59) and the output of the modified blended multiple model system, in response to the same input, is shown in figure $3 \mathrm{~b}$. The output of the modified blended multiple model system is a reasonable approximation to the output of the nonlinear system, (59), although a steady offset is still evident. In figure 4 , the output, $\widetilde{y}$, from the modified blended multiple model system is compared with the weighted linear combination of the outputs, $\breve{y}_{i}$, from the local models (with weighting $\mu_{i}(0.75)$ for the solution to the $i^{\text {th }}$ local model since the mean value of $\mathrm{x}_{1}$, for the test input considered, is approximately $0.75 \mathrm{rad}$ ). Clearly, and in accordance with the analysis in section 2, the solution to the blended multiple model system is not a straightforward blend of the solutions to the local models.

The velocity-form of the nonlinear system, (59), is

$$
\left[\begin{array}{l}
\dot{\mathrm{x}}_{1} \\
\dot{\mathrm{x}}_{2}
\end{array}\right]=\left[\begin{array}{l}
\mathrm{w}_{1} \\
\mathrm{w}_{2}
\end{array}\right], \quad\left[\begin{array}{c}
\dot{\mathrm{w}}_{1} \\
\dot{\mathrm{w}}_{2}
\end{array}\right]=\left[\begin{array}{cc}
0 & 1 \\
-\mathrm{Q} \cos \left(\mathrm{x}_{1}\right) & -\mathrm{Q}
\end{array}\right]\left[\begin{array}{l}
\mathrm{w}_{1} \\
\mathrm{w}_{2}
\end{array}\right]+\left[\begin{array}{l}
0 \\
1
\end{array}\right] \dot{\mathrm{r}}, \quad \dot{\mathrm{y}}=\left[\begin{array}{ll}
1 & 0
\end{array}\right]\left[\begin{array}{l}
\mathrm{w}_{1} \\
\mathrm{w}_{2}
\end{array}\right]
$$

and the velocity-based linearisation at an operating point, $\left(\mathrm{x}_{10}, \mathrm{x}_{20}, \mathrm{r}_{\mathrm{o}}\right)$, is obtained by simply "freezing" (64) at that operating point. A velocity-based blended multiple model system, obtained by blending the velocity-based linearisations associated with the operating points at which $\mathrm{x}_{1}$ is $0, \pi / 2$ and $\pi$, is

$$
\begin{aligned}
& {\left[\begin{array}{c}
\dot{\tilde{\mathrm{x}}}_{1} \\
\tilde{\mathrm{x}}_{2}
\end{array}\right]=\left[\begin{array}{c}
\tilde{\mathrm{w}}_{1} \\
\tilde{\mathrm{w}}_{2}
\end{array}\right]} \\
& {\left[\begin{array}{c}
\tilde{\tilde{\mathrm{w}}}_{1} \\
\tilde{\mathrm{w}}_{2}^{\prime}
\end{array}\right]=\sum_{\mathrm{i}=1}^{3}\left(\left[\begin{array}{cc}
0 & 1 \\
-\mathrm{a}_{\mathrm{i}} & -\mathrm{Q}
\end{array}\right]\left[\begin{array}{l}
\tilde{\mathrm{w}}_{1} \\
\tilde{\mathrm{w}}_{2}
\end{array}\right]+\left[\begin{array}{l}
0 \\
1
\end{array}\right] \mathrm{r}\right) \mu_{\mathrm{i}}\left(\tilde{\mathrm{x}}_{1}\right)=\left[\begin{array}{cc}
0 & 1 \\
-\sum_{\mathrm{i}=1}^{3} \mathrm{a}_{\mathrm{i}} \mu_{\mathrm{i}}\left(\tilde{\mathrm{x}}_{1}\right) & -\mathrm{Q}
\end{array}\right]\left[\begin{array}{c}
\tilde{\mathrm{w}}_{1} \\
\tilde{\mathrm{w}}_{2}
\end{array}\right]+\left[\begin{array}{l}
0 \\
1
\end{array}\right] \dot{\mathrm{r}}} \\
& \dot{\tilde{y}}=\sum_{\mathrm{i}=1}^{3}\left[\begin{array}{ll}
1 & 0
\end{array}\right]\left[\begin{array}{c}
\tilde{\mathrm{w}}_{1} \\
\tilde{\mathrm{w}}_{2}
\end{array}\right] \mu_{\mathrm{i}}\left(\widetilde{\mathrm{x}}_{1}\right)=\left[\begin{array}{ll}
1 & 0
\end{array}\right]\left[\begin{array}{c}
\tilde{\mathrm{w}}_{1} \\
\tilde{\mathrm{w}}_{2}
\end{array}\right]
\end{aligned}
$$

where $\mathrm{a}_{1}=\mathrm{Q} \cos (0), \mathrm{a}_{2}=\mathrm{Q} \cos (\pi / 2), \mathrm{a}_{3}=\mathrm{Q} \cos (\pi)$. In figure 5 , the right-hand side of the differential equation for $\tilde{\mathrm{w}}_{2}$ in the velocity-based blended multiple model system is compared to the right-hand side of the differential equation for $\mathrm{w}_{2}$ in (64) (with $\mathrm{w}_{2}$ and $\dot{\mathrm{r}}$ zero). The difference between the output of (59) and the 
output of the velocity-based blended multiple model system, in response to the same input, is depicted in figure $3 \mathrm{~b}$. It is evident that the output of the velocity-based blended multiple model system is a rather accurate approximation to the output of the nonlinear system, (59). The corresponding state solution, $\tilde{\mathrm{w}}_{2}$, to the velocity-based blended multiple model system is shown in figure $6 \mathrm{a}$. The difference between $\tilde{\mathrm{w}}_{2}$ and the weighted linear combination of the solutions to the local models is shown in figure 6b. Similar results are obtained with regard to $\tilde{\mathrm{w}}_{1}$, and it is clear that the solution to the velocity-based blended multiple model system is very closely related to the solutions to the local models, as expected from the analysis in section 3.2 .

The velocity-based blended multiple model system , (64), is depicted in figure $7 \mathrm{a}$. Of course, since pure derivative action cannot be realised, (64) cannot, in general, be realised as in figure 7a unless $\dot{\mathrm{r}}$ is available. However, by integrating, (64) may be reformulated equivalently as

$$
\left[\begin{array}{c}
\dot{\tilde{x}}_{1} \\
\dot{\tilde{x}}_{2}
\end{array}\right]=\left[\begin{array}{cc}
0 & 1 \\
0 & -\mathrm{Q}
\end{array}\right]\left[\begin{array}{c}
\tilde{\mathrm{x}}_{1} \\
\tilde{\mathrm{x}}_{2}
\end{array}\right]+\left[\begin{array}{l}
0 \\
1
\end{array}\right] \mathrm{r}+\left[\begin{array}{c}
0 \\
-\mathrm{A}\left(\tilde{\mathrm{x}}_{1}\right)
\end{array}\right], \quad \tilde{\mathrm{y}}=\left[\begin{array}{ll}
1 & 0
\end{array}\right]\left[\begin{array}{c}
\tilde{\mathrm{x}}_{1} \\
\tilde{\mathrm{x}}_{2}
\end{array}\right]
$$

where

$$
\mathrm{A}\left(\tilde{\mathrm{x}}_{1}\right)=\int_{0}^{\tilde{x}_{1}}\left(\sum_{\mathrm{i}=1}^{3} \mathrm{a}_{\mathrm{i}} \mu_{\mathrm{i}}(\mathrm{s})\right) \mathrm{ds}
$$

The realisation, (66), depicted in figure $7 \mathrm{~b}$, does not necessitate a differentiation element.

\section{Conclusions}

The blending in a blended multiple model system is required to provide smooth interpolation, in some sense, between the local models with the aim of achieving an accurate representation with only a small number of local models. The operating regions, in which no single local model dominates and several local models contribute significantly to the blended multiple model system, constitute the greater part of the operating space and it is required that dynamics of the blended multiple model system in these regions are directly related to the local models. However, it is shown that the conventional type of blended multiple model system does not meet this requirement. Furthermore, although widely employed in the literature, it is noted that affine local models do not provide continuity with established linear methods and, in particular, do not possess the superposition property which is fundamental to linear systems. The magnitude of the inhomogeneous term in an affine local model may be extremely large and can strongly influence the solution. When the inhomogeneous term varies from one local model to another, it introduces an implicit feedback from the state and/or input of the overall blended multiple model system. Hence, the inhomogeneous term cannot, in general, simply be treated as a disturbance or small error.

A novel class of velocity-based blended multiple model systems is proposed which resolves these difficulties. For blended multiple model systems in this class,

- the dynamics are directly related to the local models: the solution to a velocity-based blended multiple model system, locally to a specific operating point, is described by the solution to the linear system obtained by "freezing" the blended multiple model system at the relevant operating point. The resulting frozen system is simply a weighted linear combination of the local models.

- the solution to the blended multiple model system, locally to a specific operating point, is approximated by the weighted linear combination of the solutions to the local models.

- the local models are linear, thereby providing a degree of continuity with established linear methods and, consequently, facilitating analysis and design.

- when combined with a corresponding velocity-based blended multiple model controller, the closedloop dynamics are approximately described by the weighted combination of the closed-loop local models, thereby facilitating controller design and analysis.

These benefits stem directly from the adoption of the velocity-based linearisation framework for the analysis of nonlinear systems.

\section{Acknowledgements}


D.J. Leith acknowledges the generous support provided by the Royal Society for the work presented and the contribution from a number of stimulating discussions with Robert Shorten, University College Dublin.

\section{References}

GAWTHROP,P.J.,1995, Continuous-time local state local model networks. Proceedings of IEEE Conference on Systems, Man \& Cybernetics, Vancouver, Canada, pp852-857.

HUNT, K.J.,JOHANSEN,T.A., 1997, Design and analysis of gain-scheduled control using local controller networks. International Journal of Control, 66, 619-651.

JOHANSEN, T.A., FOSS,B.A., 1993 , Constructing NARMAX models using ARMAX models. International Journal of Control, 58, 1125-1153.

JOHANSEN, T.A., MURRAY-SMITH,R., 1997, The operating regime approach to nonlinear modelling and Control. Multiple Model Approaches to Modelling \& Control (Ed. Murray-Smith, R., Johansen, T.A.) (Taylor \& Francis, London).

KHALIL, H.K., 1992, Nonlinear Systems. (Macmillan, New York).

LEITH,D.J., LEITHEAD,W.E., 1996, Appropriate realisation of gain-scheduled controllers with application to wind turbine regulation. International Journal of Control, 65, 223-248.

LEITH,D.J., LEITHEAD,W.E., 1998a, Gain-scheduled \& nonlinear systems: dynamic analysis by velocitybased linearisation families. International Journal of Control, 70, 289-317.

LEITH,D.J., LEITHEAD,W.E., 1998b, Appropriate realisation of MIMO gain-scheduled controllers. International Journal of Control, 70, 13-50.

LEITH,D.J., LEITHEAD,W.E., 1998c, Gain-scheduled controller design: analytic framework directly incorporating non-equilibrium plant dynamics. International Journal of Control, 70, 249-269.

SHORTEN,R., MURRAY-SMITH,R.,BJORGAN,R.,GOLLEE,H.,1998, On the interpretation of local models in blended multiple model structure. International Journal of Control, submitted.

TAKAGI,T., SUGENO,M., 1985, Fuzzy identification of systems and its applications to modelling and control. IEEE Transactions on Systems, Man and Cybernetics, 15, 117-132.

TIKHONOV,A.N.M ARSENIN,V.Y.M, 1977, Solutions of Ill-posed Problems. (Wiley, New York). 


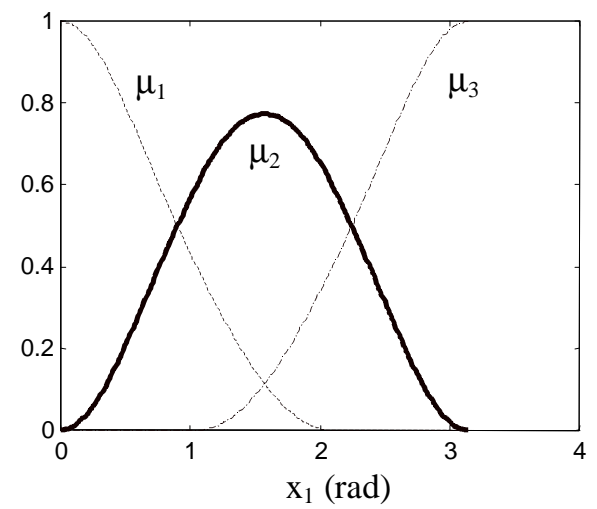

Figure 1 Validity functions used in Example.

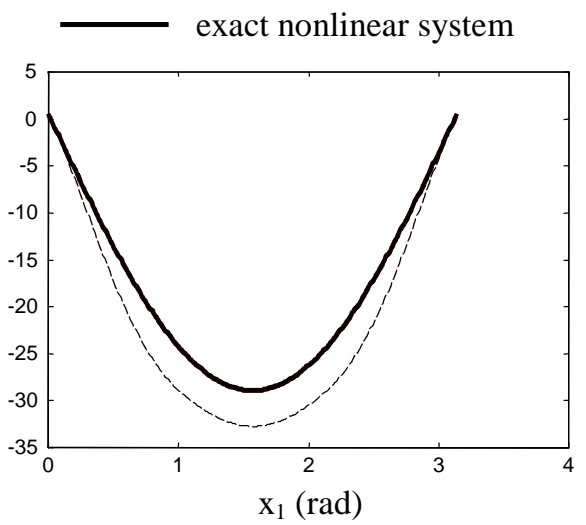

(a)
---- blended multiple model system

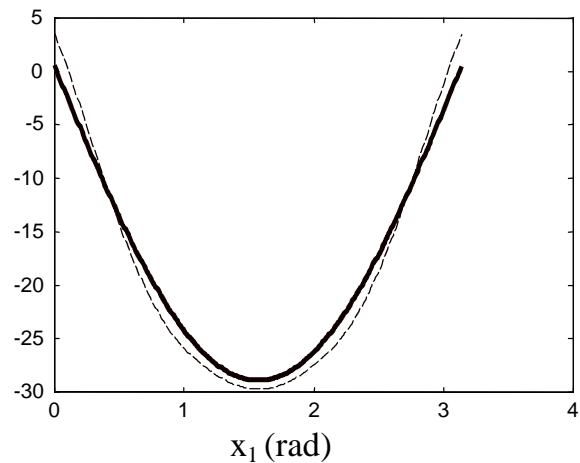

(b)

Figure 2 Fit of conventional blended multiple model system to exact nonlinear system.

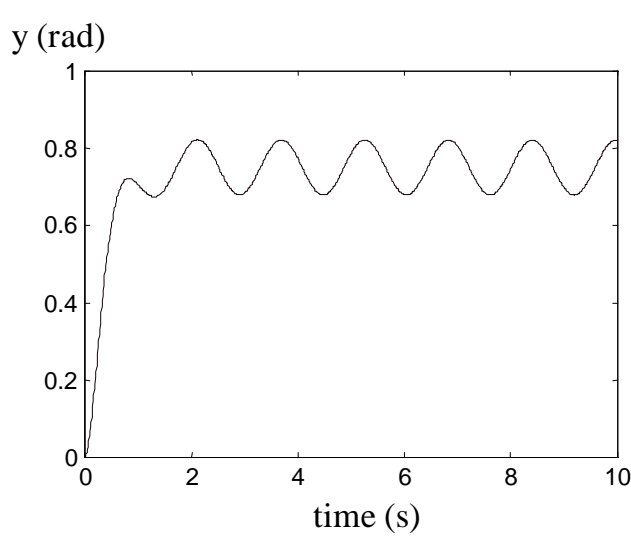

(a)

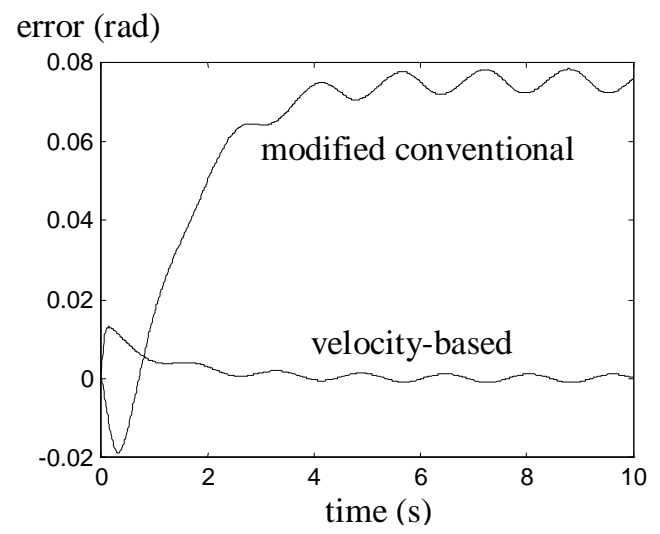

(b)

Figure 3 Output of exact nonlinear system compared with the outputs of modified conventional blended multiple model system and velocity-based multiple model system. 


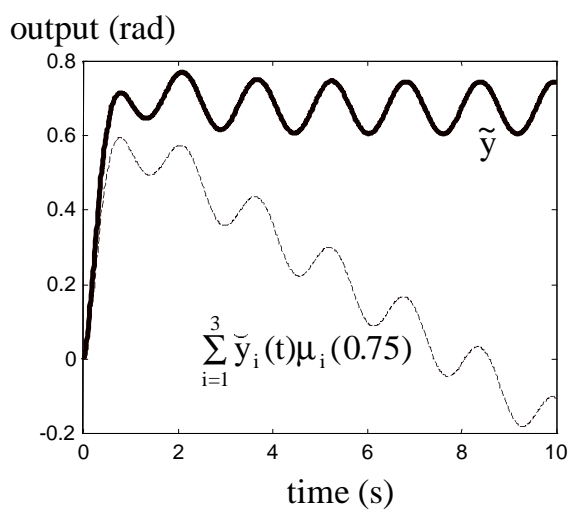

Figure 4 Output, $\tilde{y}$, from the modified conventional blended multiple model system and the weighted linear combination of the outputs, $\breve{\mathrm{y}}_{\mathrm{i}}$, from the local models.

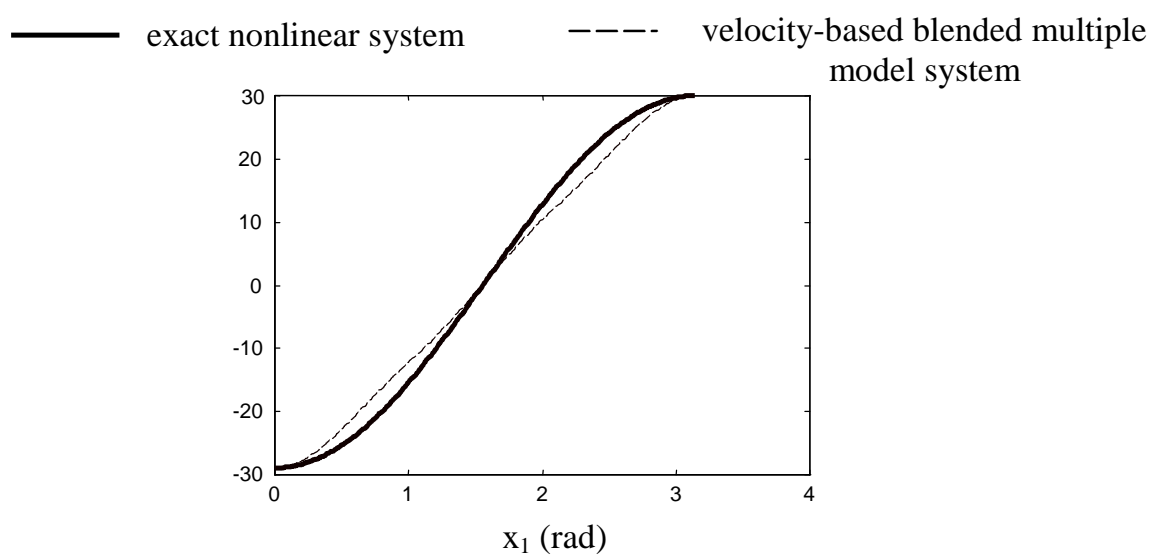

Figure 5 Fit of velocity-based blended multiple model system to exact velocity-based nonlinear system.

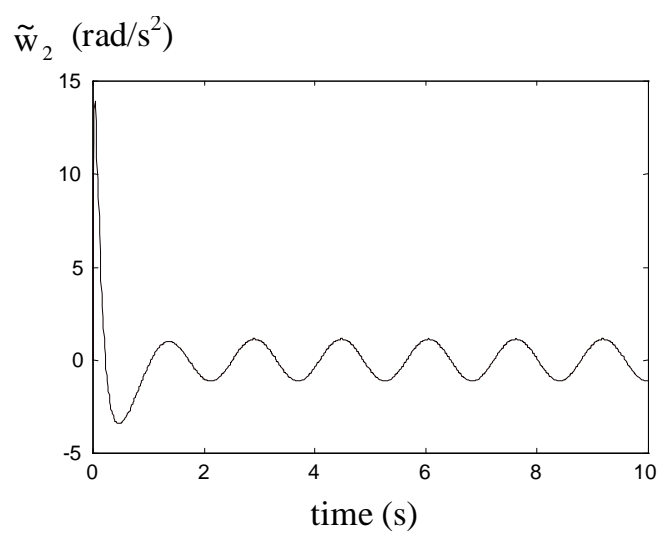

(a)

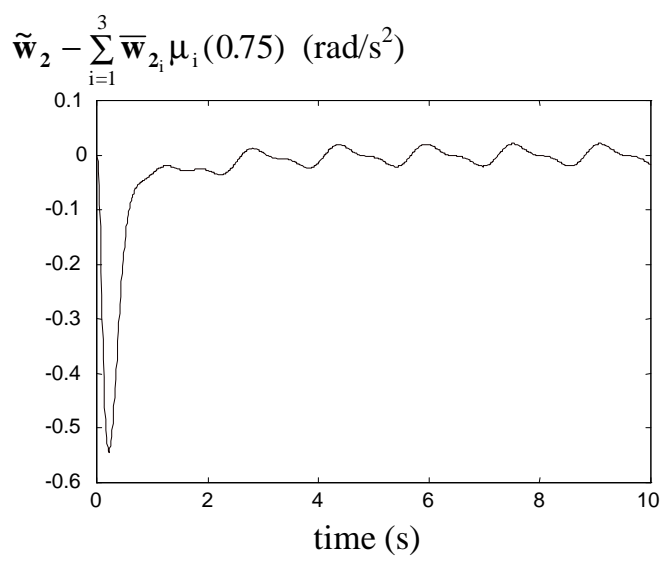

(b)

Figure 6 Solution, $\tilde{\mathrm{w}}_{2}$, to velocity-based blended multiple model system compared the weighted linear combination of the corresponding solutions, $\overline{\mathbf{w}}_{\mathbf{2}_{\mathrm{i}}}$, to local models. 


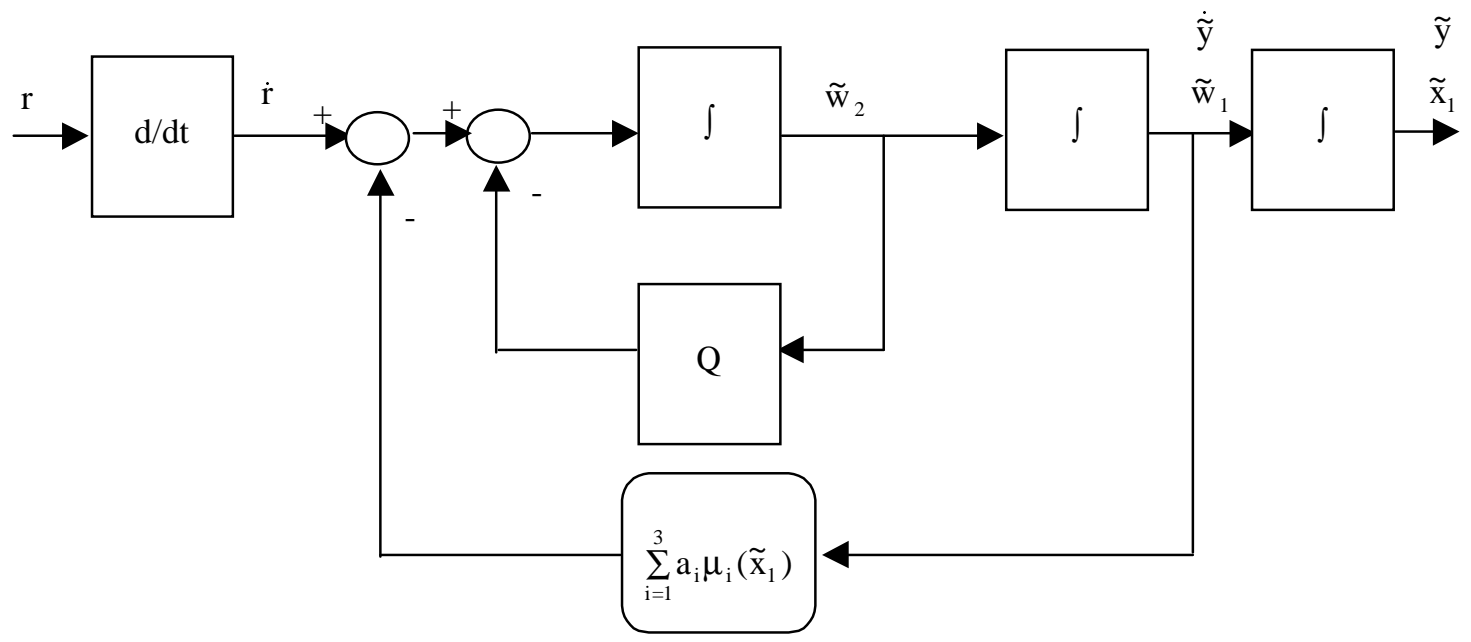

(a)

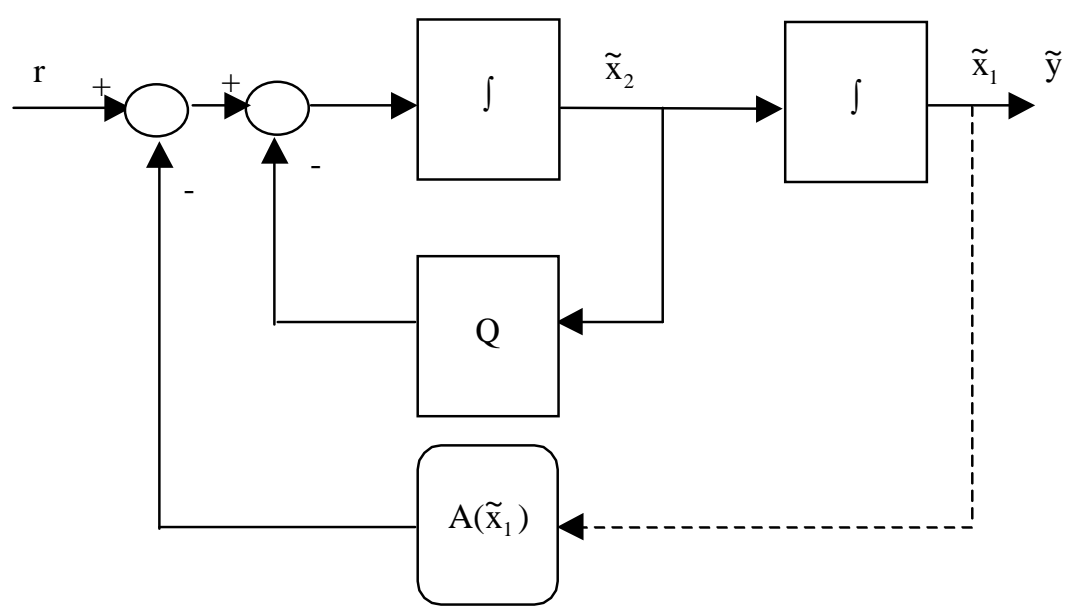

(b)

Figure 7 Realisations of velocity-based blended multiple model system. 